\title{
Anti-periodicity on high-order inertial Hopfield neural networks involving mixed delays
}

\author{
Luogen $\mathrm{YaO}^{1 *}$ (D) and Qian $\mathrm{CaO}^{2}$
}

\author{
"Correspondence: \\ yaoluogen2016@163.com \\ ${ }^{1}$ Key Laboratory of Hunan Province \\ for Statistical Learning and \\ Intelligent Computation; School of \\ Mathematics and Statistics, Hunan \\ University of Technology and \\ Business, 410205 Changsha, Hunan, \\ P.R. China \\ Full list of author information is \\ available at the end of the article
}

\begin{abstract}
This paper deals with a class of high-order inertial Hopfield neural networks involving mixed delays. Utilizing differential inequality techniques and the Lyapunov function method, we obtain a sufficient assertion to ensure the existence and global exponential stability of anti-periodic solutions of the proposed networks. Moreover, an example with a numerical simulation is furnished to illustrate the effectiveness and feasibility of the theoretical results.
\end{abstract}

Keywords: High-order inertial neural networks; Anti-periodic solution; Global exponential stability; Mixed delay

\section{Introduction}

The inertial neural networks model, which was first proposed by Babcock and Westerwelt $[1,2]$, is one of the other popular artificial neural network models used in a variety of application areas. This type of neural networks has received much attention by many researchers. In particular, numerous works have been devoted to study the dynamic behaviors on inertial neural networks with time-varying delays and some excellent results are reported, for example, stability [3-5], Hopf bifurcation [6-11], and synchronization [12-14]. To the best of our knowledge, the dynamics analysis on inertial neural networks is usually to convert them into a first-order differential system by reducing order variable substitution under the assumption that the activation functions are bounded [15-17]. However, the authors in $[12,18-21]$ pointed out that the above method not only raises the dimension in the inertial neural networks system, but also increases huge amount of computation which makes it difficult to realize in practice. For the above reasons, the authors of $[19,20]$ and [21], respectively, developed some non-reduced order techniques to investigate the stability and synchronization of inertial neural networks with different types of time delays.

On the other hand, in neural networks dynamics involving fields such as communication, economics, biology or ecology, the relevant state variables are usually considered as proteins and molecules, light intensity levels or electric charges, which are naturally antiperiodic [22-24]. Considering this factor, many recurrent neural network models involv-

(c) The Author(s) 2020. This article is licensed under a Creative Commons Attribution 4.0 International License, which permits use sharing, adaptation, distribution and reproduction in any medium or format, as long as you give appropriate credit to the original author(s) and the source, provide a link to the Creative Commons licence, and indicate if changes were made. The images or other third party material in this article are included in the article's Creative Commons licence, unless indicated otherwise in a credit line to the material. If material is not included in the article's Creative Commons licence and your intended use is not permitted by statutory regulation or exceeds the permitted use, you will need to obtain permission directly from the copyright holder. To view a copy of this licence, visit http://creativecommons.org/licenses/by/4.0/. 
ing time-varying delays and anti-periodic environments have been widely investigated in $[16,17,24-26]$. It is worth noting that the high-order Hopfield neural networks have the advantages of faster convergence speed, larger storage capacity and stronger fault tolerance than lower-order neural networks [27-29]. Consequently, Yao [30] studied the existence and global exponential stability of anti-periodic solutions for a class of proportional delayed high-order inertial Hopfield neural networks with time-varying delays.

In recent years, the authors in [21] have mentioned that many parallel routes with a series of different axon sizes and lengths appear in neural networks, and it is desired to explain the dynamics behaviors of neural networks by involving continuously distributed delays. Furthermore, the dynamic behaviors of many recurrent neural networks with continuously distributed delays have been revealed in [27, 31-35]. However, few articles have considered the anti-periodic problem for the following high-order inertial Hopfield neural networks (HIHNNs) involving time-varying delays and continuously distributed delays:

$$
\begin{aligned}
x_{i}^{\prime \prime}(t)=- & a_{i}(t) x_{i}^{\prime}(t)-b_{i}(t) x_{i}(t)+\sum_{j=1}^{n} c_{i j}(t) A_{j}\left(x_{j}(t)\right) \\
& +\sum_{j=1}^{n} d_{i j}(t) B_{j}\left(x_{j}\left(t-q_{i j}(t)\right)\right) \\
& +\sum_{j=1}^{n} \sum_{l=1}^{n} \theta_{i j l}(t) Q_{j}\left(x_{j}\left(t-\eta_{i j l}(t)\right)\right) Q_{l}\left(x_{l}\left(t-\xi_{i j l}(t)\right)\right) \\
& +\sum_{j=1}^{n} h_{i j}(t) \int_{0}^{+\infty} \sigma_{i j}(u) K_{j}\left(x_{j}(t-u)\right) d u \\
& +\sum_{j=1}^{n} \sum_{l=1}^{n} p_{i j l}(t) \int_{0}^{+\infty} \hat{\sigma}_{i j l}(u) R_{j}\left(x_{j}(t-u)\right) d u \\
& \times \int_{0}^{+\infty} \bar{\sigma}_{i j l}(u) R_{l}\left(x_{l}(t-u)\right) d u+J_{i}(t),
\end{aligned}
$$

and the initial value conditions:

$$
x_{i}(s)=\varphi_{i}(s), \quad x_{i}^{\prime}(0)=\psi_{i}, \quad-\infty \leq s \leq 0, \varphi_{i} \in B C((-\infty, 0], \mathbb{R}), \psi_{i} \in \mathbb{R},
$$

where $B C((-\infty, 0], \mathbb{R})$ is the set of all continuous and bounded functions from $(-\infty, 0]$ to $\mathbb{R}, J_{i}, c_{i j}, d_{i j}, \theta_{i j l}, h_{i j}, p_{i j l}: \mathbb{R} \rightarrow \mathbb{R}, a_{i}, b_{i}: \mathbb{R} \rightarrow(0,+\infty)$ and $q_{i j}, \eta_{i j l}, \xi_{i j l}: \mathbb{R} \rightarrow \mathbb{R}^{+}$are bounded and continuous functions, $a_{i}, b_{i}, q_{i j}, \eta_{i j l}, \xi_{i j l}$ are periodic functions with period $T>0$, the input term $J_{i}$ is $T$-anti-periodic (i.e. $J_{i}(t+T)=-J_{i}(t)$ for all $t \in \mathbb{R}$ ), and $i, j, l \in D=\{1,2, \ldots, n\}$.

Motivated by the previous discussions, in this paper, without adopting the reduced order method, we shall install new results concerning the anti-periodic dynamics for HIHNNs with time-varying delays and continuously distributed delays. Some sufficient conditions ensuring the existence and global exponential stability on the anti-periodic solution of system (1.1) are established by using differential inequalities and the Lyapunov function method, which improve and complement some earlier publications [16, 17, 36-40].

We organize the paper as follows. In Sect. 2, some assumptions and an important lemmas are listed. Section 3 presents the main results and their detailed proof. Section 4 gives 
a numerical example to demonstrate the feasibility of the main results. Conclusions are drawn in Sect. 5.

\section{Preliminary results}

In this section, some assumptions and a key lemma are provided.

\section{Assumptions}

$\left(G_{1}\right)$ There are nonnegative constants $L_{j}^{A}, L_{j}^{B}, L_{j}^{Q}, L_{j}^{K}, L_{j}^{R}, M_{j}^{Q}$ and $M_{j}^{R}$ such that

$$
\begin{aligned}
& \left\{\begin{array}{l}
\left|A_{j}(u)-A_{j}(v)\right| \leq L_{j}^{A}|u-v|, \quad\left|B_{j}(u)-B_{j}(v)\right| \leq L_{j}^{B}|u-v|, \\
\left|Q_{j}(u)-Q_{j}(v)\right| \leq L_{j}^{Q}|u-v|, \quad\left|K_{j}(u)-K_{j}(v)\right| \leq L_{j}^{K}|u-v|, \\
\left|R_{j}(u)-R_{j}(v)\right| \leq L_{j}^{R}|u-v|, \quad\left|Q_{j}(u)\right| \leq M_{j}^{Q}, \quad\left|R_{j}(u)\right| \leq M_{j}^{R},
\end{array}\right. \\
& \left\{\begin{array}{l}
c_{i j}(t+T) A_{j}(u)=-c_{i j}(t) A_{j}(-u), \quad d_{i j}(t+T) B_{j}(u)=-d_{i j}(t) B_{j}(-u), \\
\theta_{i j l}(t+T) Q_{j}(u) Q_{l}(v)=-\theta_{i j l}(t) Q_{j}(-u) Q_{l}(-v), \\
h_{i j}(t+T) \int_{0}^{+\infty} \sigma_{i j}(s) K_{j}(u) d s=-h_{i j}(t) \int_{0}^{+\infty} \sigma_{i j}(s) K_{j}(-u) d s,
\end{array}\right.
\end{aligned}
$$

and

$$
\begin{aligned}
& p_{i j l}(t+T) \int_{0}^{+\infty} \hat{\sigma}_{i j l}(s) R_{j}(u) d s \int_{0}^{+\infty} \bar{\sigma}_{i j l}(s) R_{l}(u) d s \\
& =-p_{i j l}(t) \int_{0}^{+\infty} \hat{\sigma}_{i j l}(s) R_{j}(-u) d s \int_{0}^{+\infty} \bar{\sigma}_{i j l}(s) R_{l}(-u) d s,
\end{aligned}
$$

for all $u, v \in \mathbb{R}, i, j, l \in D$.

$\left(G_{2}\right)$ For $i, j, l \in D,\left|\sigma_{i j}(t)\right| e^{\mu t},\left|\hat{\sigma}_{i j l}(t)\right| e^{\mu t},\left|\bar{\sigma}_{i j l}(t)\right| e^{\mu t}$ are integrable on $[0,+\infty)$ for a positive constant $\mu$.

$\left(G_{3}\right)$ There are constants $\beta_{i}>0$ and $\alpha_{i} \geq 0, \gamma_{i} \geq 0$ obeying

$$
C_{i}(t)<0, \quad 4 C_{i}(t) D_{i}(t)>E_{i}^{2}(t), \quad \forall t \in \mathbb{R}, i \in D,
$$

where

$$
\begin{aligned}
C_{i}(t)= & \alpha_{i} \gamma_{i}-a_{i}(t) \alpha_{i}^{2}+\frac{1}{2} \alpha_{i}^{2} \sum_{j=1}^{n}\left(\left|c_{i j}(t)\right| L_{j}^{A}+\left|d_{i j}(t)\right| L_{j}^{B}\right) \\
& +\frac{1}{2} \alpha_{i}^{2} \sum_{j=1}^{n} \sum_{l=1}^{n}\left|\theta_{i j l}(t)\right|\left(M_{j}^{Q} L_{l}^{Q}+L_{j}^{Q} M_{l}^{Q}\right) \\
& +\frac{1}{2} \alpha_{i}^{2} \sum_{j=1}^{n}\left|h_{i j}(t)\right| L_{j}^{K} \int_{0}^{+\infty}\left|\sigma_{i j}(u)\right| d u \\
& +\frac{1}{2} \alpha_{i}^{2} \sum_{j=1}^{n} \sum_{l=1}^{n}\left|p_{i j l}(t)\right| M_{j}^{R} L_{l}^{R} \int_{0}^{+\infty}\left|\hat{\sigma}_{i j l}(u)\right| d u \int_{0}^{+\infty}\left|\bar{\sigma}_{i j l}(u)\right| d u \\
& +\frac{1}{2} \alpha_{i}^{2} \sum_{j=1}^{n} \sum_{l=1}^{n}\left|p_{i j l}(t)\right| M_{l}^{R} L_{j}^{R} \int_{0}^{+\infty}\left|\bar{\sigma}_{i j l}(u)\right| d u \int_{0}^{+\infty}\left|\hat{\sigma}_{i j l}(u)\right| d u,
\end{aligned}
$$




$$
\begin{aligned}
& D_{i}(t)=-b_{i}(t) \alpha_{i} \gamma_{i}+\frac{1}{2} \sum_{j=1}^{n}\left(\left|c_{i j}(t)\right| L_{j}^{A}+\left|d_{i j}(t)\right| L_{j}^{B}\right)\left|\alpha_{i} \gamma_{i}\right| \\
& +\frac{1}{2} \sum_{j=1}^{n} \alpha_{j}^{2}\left(\left|c_{j i}(t)\right| L_{i}^{A}+d_{j i}^{+} L_{i}^{B} \frac{1}{1-\dot{q}_{j i}^{+}} e^{2 \lambda q_{j i}^{+}}\right) \\
& +\frac{1}{2} \sum_{j=1}^{n}\left(\left|c_{j i}(t)\right| L_{i}^{A}+d_{j i}^{+} L_{i}^{B} \frac{1}{1-\dot{q}_{j i}^{+}} e^{2 \lambda q_{j i}^{+}}\right)\left|\alpha_{j} \gamma_{j}\right| \\
& +\frac{1}{2} \sum_{j=1}^{n} \sum_{l=1}^{n}\left|\alpha_{i} \gamma_{i}\right|\left|\theta_{i j l}(t)\right|\left(M_{j}^{Q} L_{l}^{Q}+L_{j}^{Q} M_{l}^{Q}\right) \\
& +\frac{1}{2} \sum_{l=1}^{n} \sum_{j=1}^{n}\left(\alpha_{l}^{2}+\left|\alpha_{l} \gamma_{l}\right|\right) \theta_{l j i}^{+} M_{j}^{Q} L_{i}^{Q} e^{2 \lambda \xi_{l j i}^{+}} \frac{1}{1-\dot{\xi}_{l j i}^{+}} \\
& +\frac{1}{2} \sum_{j=1}^{n} \sum_{l=1}^{n}\left(\alpha_{j}^{2}+\left|\alpha_{j} \gamma_{j}\right|\right) \theta_{j i l}^{+} L_{i}^{Q} M_{l}^{Q} e^{2 \lambda \eta_{j i l}^{+}} \frac{1}{1-\dot{\eta}_{j i l}^{+}} \\
& +\frac{1}{2} \sum_{j=1}^{n}\left(\alpha_{j}^{2} h_{j i}^{+}+\left|\alpha_{j} \gamma_{j}\right| h_{j i}^{+}\right) L_{i}^{K} \int_{0}^{+\infty}\left|\sigma_{j i}(u)\right| e^{2 \lambda u} d u \\
& +\frac{1}{2} \sum_{l=1}^{n} \sum_{j=1}^{n}\left(\alpha_{l}^{2}+\left|\alpha_{l} \gamma_{l}\right|\right) p_{l j i}^{+} \int_{0}^{+\infty}\left|\hat{\sigma}_{l j i}(u)\right| d u \int_{0}^{+\infty}\left|\bar{\sigma}_{l j i}(u)\right| e^{2 \lambda u} d u M_{j}^{R} L_{i}^{R} \\
& +\frac{1}{2} \sum_{j=1}^{n} \sum_{l=1}^{n}\left(\alpha_{j}^{2}+\left|\alpha_{j} \gamma_{j}\right|\right) p_{j i l}^{+} \int_{0}^{+\infty}\left|\bar{\sigma}_{j i l}(u)\right| d u \int_{0}^{+\infty}\left|\hat{\sigma}_{j i l}(u)\right| e^{2 \lambda u} d u M_{l}^{R} L_{i}^{R} \\
& +\frac{1}{2} \sum_{j=1}^{n}\left|\alpha_{i} \gamma_{i}\right|\left|h_{i j}(t)\right| L_{j}^{K} \int_{0}^{+\infty}\left|\sigma_{i j}(u)\right| d u \\
& +\frac{1}{2} \sum_{j=1}^{n} \sum_{l=1}^{n}\left|\alpha_{i} \gamma_{i}\right|\left|p_{i j l}(t)\right| M_{j}^{R} L_{l}^{R} \int_{0}^{+\infty}\left|\hat{\sigma}_{i j l}(u)\right| d u \int_{0}^{+\infty}\left|\bar{\sigma}_{i j l}(u)\right| d u \\
& +\frac{1}{2} \sum_{j=1}^{n} \sum_{l=1}^{n}\left|\alpha_{i} \gamma_{i}\right|\left|p_{i j l}(t)\right| M_{l}^{R} L_{j}^{R} \int_{0}^{+\infty}\left|\bar{\sigma}_{i j l}(u)\right| d u \int_{0}^{+\infty}\left|\hat{\sigma}_{i j l}(u)\right| d u, \\
& E_{i}(t)=\beta_{i}+\gamma_{i}^{2}-a_{i}(t) \alpha_{i} \gamma_{i}-b_{i}(t) \alpha_{i}^{2}, \quad \dot{q}_{i j}^{+}=\max _{t \in[0, T]} q_{i j}^{\prime}(t), \\
& \dot{\eta}_{i j l}^{+}=\max _{t \in[0, T]} \eta_{i j l}^{\prime}(t), \quad \dot{\xi}_{i j l}^{+}=\max _{t \in[0, T]} \xi_{i j l}^{\prime}(t), \\
& q_{i j}^{+}=\max _{t \in[0, T]} q_{i j}(t), \quad \eta_{i j l}^{+}=\max _{t \in[0, T]} \eta_{i j l}(t),
\end{aligned}
$$

and

$$
\begin{aligned}
& \xi_{i j l}^{+}=\max _{t \in[0, T]} \xi_{i j l}(t), \quad c_{i j}^{+}=\sup _{t \in \mathbb{R}}\left|c_{i j}(t)\right|, \quad d_{i j}^{+}=\sup _{t \in \mathbb{R}}\left|d_{i j}(t)\right|, \\
& \theta_{i j l}^{+}=\sup _{t \in \mathbb{R}}\left|\theta_{i j l}(t)\right|, \quad i, j, l \in D .
\end{aligned}
$$

$\left(G_{4}\right)$ For $i, j, l \in D, q_{i j}, \eta_{i j l}$ and $\xi_{i j l}$ are continuously differentiable, $q_{i j}^{\prime}(t)=\dot{q}_{i j}(t)<1, \eta_{i j l}^{\prime}(t)=$ $\eta_{i j l}(t)<1$ and $\xi_{i j l}^{\prime}(t)=\dot{\xi_{i j l}}(t)<1$, for all $t \in \mathbb{R}$. 
Remark 2.1 According to $\left(G_{1}\right)$ and the basic theory on functional differential equation with infinite delay in [41], one can show that all solutions of (1.1) and (1.2) exist in $[0,+\infty)$.

Lemma 2.1 Assume that $\left(G_{1}\right),\left(G_{2}\right),\left(G_{3}\right)$ and $\left(G_{4}\right)$ hold. Let $x(t)=\left(x_{1}(t), x_{2}(t), \ldots, x_{n}(t)\right)$ and $y(t)=\left(y_{1}(t), y_{2}(t), \ldots, y_{n}(t)\right)$ be two solutions of system (1.1) satisfying

$$
x_{i}(s)=\varphi_{i}^{x}(s), \quad x_{i}^{\prime}(0)=\psi_{i}^{x}, \quad y_{i}(s)=\varphi_{i}^{y}(s), \quad y_{i}^{\prime}(0)=\psi_{i}^{y},
$$

where $i \in D, \varphi_{i}^{x}, \varphi_{i}^{y} \in B C((-\infty, 0], \mathbb{R}), \psi_{i}^{x}, \psi_{i}^{y} \in \mathbb{R}$. Then there are two positive constants $\lambda$ and $M=M\left(\varphi^{x}, \psi^{x}, \varphi^{y}, \psi^{y}\right)$ such that

$$
\left|x_{i}(t)-y_{i}(t)\right| \leq M e^{-\lambda t}, \quad\left|x_{i}^{\prime}(t)-y_{i}^{\prime}(t)\right| \leq M e^{-\lambda t}, \quad \text { for all } t \geq 0, i \in D \text {. }
$$

Proof Denote $z_{i}(t)=y_{i}(t)-x_{i}(t)$, then

$$
\begin{aligned}
z_{i}^{\prime \prime}(t) & \\
= & -a_{i}(t) z_{i}^{\prime}(t)-b_{i}(t) z_{i}(t) \\
& +\sum_{j=1}^{n} c_{i j}(t) \widetilde{A}_{j}\left(z_{j}(t)\right)+\sum_{j=1}^{n} d_{i j}(t) \widetilde{B}_{j}\left(z_{j}\left(t-q_{i j}(t)\right)\right)+\sum_{j=1}^{n} \sum_{l=1}^{n} \theta_{i j l}(t) \\
& \times\left[Q_{j}\left(y_{j}\left(t-\eta_{i j l}(t)\right)\right) Q_{l}\left(y_{l}\left(t-\xi_{i j l}(t)\right)\right)-Q_{j}\left(y_{j}\left(t-\eta_{i j l}(t)\right)\right) Q_{l}\left(x_{l}\left(t-\xi_{i j l}(t)\right)\right)\right. \\
& \left.+Q_{j}\left(y_{j}\left(t-\eta_{i j l}(t)\right)\right) Q_{l}\left(x_{l}\left(t-\xi_{i j l}(t)\right)\right)-Q_{j}\left(x_{j}\left(t-\eta_{i j l}(t)\right)\right) Q_{l}\left(x_{l}\left(t-\xi_{i j l}(t)\right)\right)\right] \\
& +\sum_{j=1}^{n} h_{i j}(t) \int_{0}^{+\infty} \sigma_{i j}(u) \widetilde{K}_{j}\left(z_{j}(t-u)\right) d u+\sum_{j=1}^{n} \sum_{l=1}^{n} p_{i j l}(t) \\
& \times\left[\int_{0}^{+\infty} \hat{\sigma}_{i j l}(u) R_{j}\left(y_{j}(t-u)\right) d u \int_{0}^{+\infty} \bar{\sigma}_{i j l}(u) R_{l}\left(y_{l}(t-u)\right) d u\right. \\
& -\int_{0}^{+\infty} \hat{\sigma}_{i j l}(u) R_{j}\left(y_{j}(t-u)\right) d u \int_{0}^{+\infty} \bar{\sigma}_{i j l}(u) R_{l}\left(x_{l}(t-u)\right) d u \\
& +\int_{0}^{+\infty} \hat{\sigma}_{i j l}(u) R_{j}\left(y_{j}(t-u)\right) d u \int_{0}^{+\infty} \bar{\sigma}_{i j l}(u) R_{l}\left(x_{l}(t-u)\right) d u \\
& \left.-\int_{0}^{+\infty} \hat{\sigma}_{i j l}(u) R_{j}\left(x_{j}(t-u)\right) d u \int_{0}^{+\infty} \bar{\sigma}_{i j l}(u) R_{l}\left(x_{l}(t-u)\right) d u\right],
\end{aligned}
$$

where

$$
\left\{\begin{array}{l}
\widetilde{A}_{j}\left(z_{j}(t)\right)=A_{j}\left(y_{j}(t)\right)-A_{j}\left(x_{j}(t)\right), \\
\widetilde{B}_{j}\left(z_{j}\left(t-q_{i j}(t)\right)\right)=B_{j}\left(y_{j}\left(t-q_{i j}(t)\right)\right)-B_{j}\left(x_{j}\left(t-q_{i j}(t)\right)\right), \\
\widetilde{K}_{j}\left(z_{j}(t-u)\right)=K_{j}\left(y_{j}(t-u)\right)-K_{j}\left(x_{j}(t-u)\right),
\end{array}\right.
$$

and $i, j \in D$.

According to $\left(G_{2}\right)$ and the boundedness of (1.1), one can select a constant $\lambda>0$ such that

$$
C_{i}^{\lambda}(t)<0, \quad 4 C_{i}^{\lambda}(t) D_{i}^{\lambda}(t)>\left(E_{i}^{\lambda}(t)\right)^{2}, \quad \forall t \in \mathbb{R},
$$


where

$$
\begin{aligned}
C_{i}^{\lambda}(t)= & \lambda \alpha_{i}^{2}+\alpha_{i} \gamma_{i}-a_{i}(t) \alpha_{i}^{2}+\frac{1}{2} \alpha_{i}^{2} \sum_{j=1}^{n}\left(\left|c_{i j}(t)\right| L_{j}^{A}+\left|d_{i j}(t)\right| L_{j}^{B}\right) \\
& +\frac{1}{2} \alpha_{i}^{2} \sum_{j=1}^{n} \sum_{l=1}^{n}\left|\theta_{i j l}(t)\right|\left(M_{j}^{Q} L_{l}^{Q}+L_{j}^{Q} M_{l}^{Q}\right) \\
& +\frac{1}{2} \alpha_{i}^{2} \sum_{j=1}^{n}\left|h_{i j}(t)\right| L_{j}^{K} \int_{0}^{+\infty}\left|\sigma_{i j}(u)\right| d u \\
& +\frac{1}{2} \alpha_{i}^{2} \sum_{j=1}^{n} \sum_{l=1}^{n}\left|p_{i j l}(t)\right| M_{j}^{R} L_{l}^{R} \int_{0}^{+\infty}\left|\hat{\sigma}_{i j l}(u)\right| d u \int_{0}^{+\infty}\left|\bar{\sigma}_{i j l}(u)\right| d u \\
& +\frac{1}{2} \alpha_{i}^{2} \sum_{j=1}^{n} \sum_{l=1}^{n}\left|p_{i j l}(t)\right| M_{l}^{R} L_{j}^{R} \int_{0}^{+\infty}\left|\bar{\sigma}_{i j l}(u)\right| d u \int_{0}^{+\infty}\left|\hat{\sigma}_{i j l}(u)\right| d u,
\end{aligned}
$$

$D_{i}^{\lambda}(t)$

$$
\begin{aligned}
= & -b_{i}(t) \alpha_{i} \gamma_{i}+\lambda \beta_{i}+\lambda \gamma_{i}^{2}+\frac{1}{2} \sum_{j=1}^{n}\left(\left|c_{i j}(t)\right| L_{j}^{A}+\left|d_{i j}(t)\right| L_{j}^{B}\right)\left|\alpha_{i} \gamma_{i}\right| \\
& +\frac{1}{2} \sum_{j=1}^{n} \alpha_{j}^{2}\left(\left|c_{j i}(t)\right| L_{i}^{A}+d_{j i}^{+} L_{i}^{B} \frac{1}{1-\dot{q}_{j i}^{+}} e^{2 \lambda \gamma_{j i}^{+}}\right) \\
& +\frac{1}{2} \sum_{j=1}^{n}\left(\left|c_{j i}(t)\right| L_{i}^{A}+d_{j i}^{+} L_{i}^{B} \frac{1}{1-\dot{q}_{j i}^{+}} e^{2 \lambda q_{j i}^{+}}\right)\left|\alpha_{j} \gamma_{j}\right| \\
& +\frac{1}{2} \sum_{j=1}^{n} \sum_{l=1}^{n}\left|\alpha_{i} \gamma_{i}\right|\left|\theta_{i j l}(t)\right|\left(M_{j}^{Q} L_{l}^{Q}+L_{j}^{Q} M_{l}^{Q}\right) \\
& +\frac{1}{2} \sum_{l=1}^{n} \sum_{j=1}^{n}\left(\alpha_{l}^{2}+\left|\alpha_{l} \gamma_{l}\right|\right) \theta_{l j i}^{+} M_{j}^{Q} L_{i}^{Q} e^{2 \lambda \xi_{l j i}^{+}} \frac{1}{1-\dot{\xi}_{l j i}^{+}} \\
& +\frac{1}{2} \sum_{j=1}^{n} \sum_{l=1}^{n}\left(\alpha_{j}^{2}+\left|\alpha_{j} \gamma_{j}\right|\right) \theta_{j i l}^{+} L_{i}^{Q} M_{l}^{Q} e^{2 \lambda \eta_{j i l}^{+}} \frac{1}{1-\dot{\eta}_{j i l}^{+}} \\
& +\frac{1}{2} \sum_{j=1}^{n}\left(\alpha_{j}^{2} h_{j i}^{+}+\left|\alpha_{j} \gamma_{j}\right| h_{j i}^{+}\right) L_{i}^{K} \int_{0}^{+\infty}\left|\sigma_{j i}(u)\right| e^{2 \lambda u} d u \\
& +\frac{1}{2} \sum_{l=1}^{n} \sum_{j=1}^{n}\left(\alpha_{l}^{2}+\left|\alpha_{l} \gamma_{l}\right|\right) p_{l j i}^{+} \int_{0}^{+\infty}\left|\hat{\sigma}_{l j i}(u)\right| d u \int_{0}^{+\infty}\left|\bar{\sigma}_{l j i}(u)\right| e^{2 \lambda u} d u M_{j}^{R} L_{i}^{R} \\
& +\frac{1}{2} \sum_{j=1}^{n} \sum_{l=1}^{n}\left|\alpha_{i} \gamma_{i}\right|\left|p_{i j l}(t)\right| M_{l}^{R} L_{j}^{R} \int_{0}^{+\infty}\left|\bar{\sigma}_{i j l}(u)\right| d u \int_{0}^{+\infty}\left|\hat{\sigma}_{i j l}(u)\right| d u, \\
& +\frac{1}{2} \sum_{j=1}^{n} \sum_{l=1}^{n}\left(\alpha_{j}^{2}+\left|\alpha_{j} \gamma_{j}\right|\right) p_{j i l}^{+} \int_{0}^{+\infty}\left|\bar{\sigma}_{j i l}(u)\right| d u \int_{0}^{+\infty}\left|\hat{\sigma}_{j i l}(u)\right| e^{2 \lambda u} d u M_{l}^{R} L_{i}^{R} \\
& +\frac{1}{2} \sum_{j=1}^{n}\left|\alpha_{i} \gamma_{i}\right|\left|h_{i j}(t)\right| L_{j}^{K} \int_{0}^{+\infty}\left|\sigma_{i j}(u)\right| d u \\
& \frac{1}{2} \gamma_{i}|| p_{i j l}(t)\left|M_{j}^{R} L_{l}^{R} \int_{0}^{+\infty}\right| \hat{\sigma}_{i j l}(u)\left|d u \int_{0}^{+\infty}\right| \bar{\sigma}_{i j l}(u) \mid d u \\
&
\end{aligned}
$$


and

$$
E_{i}^{\lambda}(t)=\beta_{i}+\gamma_{i}^{2}+2 \lambda \alpha_{i} \gamma_{i}-a_{i}(t) \alpha_{i} \gamma_{i}-b_{i}(t) \alpha_{i}^{2}, \quad i \in D
$$

Set

$W(t)$

$$
\begin{aligned}
& =\frac{1}{2} \sum_{i=1}^{n} \beta_{i} z_{i}^{2}(t) e^{2 \lambda t}+\frac{1}{2} \sum_{i=1}^{n}\left(\alpha_{i} z_{i}^{\prime}(t)+\gamma_{i} z_{i}(t)\right)^{2} e^{2 \lambda t} \\
& +\frac{1}{2} \sum_{i=1}^{n} \sum_{j=1}^{n}\left(\alpha_{i}^{2} d_{i j}^{+}+\left|\alpha_{i} \gamma_{i}\right| d_{i j}^{+}\right) e^{2 \lambda q_{i j}^{+}} L_{j}^{B} \int_{t-q_{i j}(t)}^{t} z_{j}^{2}(s) \frac{1}{1-\dot{q}_{i j}^{+}} e^{2 \lambda s} d s \\
& +\frac{1}{2} \sum_{i=1}^{n} \sum_{l=1}^{n} \sum_{j=1}^{n}\left(\alpha_{l}^{2}+\left|\alpha_{l} \gamma_{l}\right|\right) \theta_{l j i}^{+} M_{j}^{Q} L_{i}^{Q} e^{2 \lambda \xi_{l j i}^{+}} \int_{t-\xi_{l j i}(t)}^{t} z_{i}^{2}(s) \frac{1}{1-\dot{\xi}_{l j i}^{+}} e^{2 \lambda s} d s \\
& +\frac{1}{2} \sum_{i=1}^{n} \sum_{j=1}^{n} \sum_{l=1}^{n}\left(\alpha_{j}^{2}+\left|\alpha_{j} \gamma_{j}\right|\right) \theta_{j i l}^{+} L_{i}^{Q} M_{l}^{Q} e^{2 \lambda \eta_{j i l}^{+}} \int_{t-\eta_{j i l}(t)}^{t} z_{i}^{2}(s) \frac{1}{1-\dot{\eta}_{j i l}^{+}} e^{2 \lambda s} d s \\
& +\frac{1}{2} \sum_{i=1}^{n} \sum_{j=1}^{n}\left(\alpha_{i}^{2} h_{i j}^{+}+\left|\alpha_{i} \gamma_{i}\right| h_{i j}^{+}\right) L_{j}^{K} \int_{0}^{+\infty}\left|\sigma_{i j}(u)\right| \int_{t-u}^{t} z_{j}^{2}(s) e^{2 \lambda(u+s)} d s d u \\
& +\frac{1}{2} \sum_{i=1}^{n} \sum_{l=1}^{n} \sum_{j=1}^{n}\left(\alpha_{l}^{2}+\left|\alpha_{l} \gamma_{l}\right|\right) p_{l j i}^{+} M_{j}^{R} L_{i}^{R} \int_{0}^{+\infty}\left|\hat{\sigma}_{l j i}(u)\right| d u \\
& \times \int_{0}^{+\infty}\left|\bar{\sigma}_{l j i}(u)\right| \int_{t-u}^{t} z_{i}^{2}(s) e^{2 \lambda(u+s)} d s d u \\
& +\frac{1}{2} \sum_{i=1}^{n} \sum_{j=1}^{n} \sum_{l=1}^{n}\left(\alpha_{j}^{2}+\left|\alpha_{j} \gamma_{j}\right|\right) p_{j i l}^{+} M_{l}^{R} L_{i}^{R} \int_{0}^{+\infty}\left|\bar{\sigma}_{j i l}(u)\right| d u \\
& \times \int_{0}^{+\infty}\left|\hat{\sigma}_{j i l}(u)\right| \int_{t-u}^{t} z_{i}^{2}(s) e^{2 \lambda(u+s)} d s d u .
\end{aligned}
$$

A straightforward computation yields

$W^{\prime}(t)$

$$
\begin{aligned}
= & 2 \lambda\left[\frac{1}{2} \sum_{i=1}^{n} \beta_{i} z_{i}^{2}(t) e^{2 \lambda t}+\frac{1}{2} \sum_{i=1}^{n}\left(\alpha_{i} z_{i}^{\prime}(t)+\gamma_{i} z_{i}(t)\right)^{2} e^{2 \lambda t}\right] \\
& +\sum_{i=1}^{n}\left(\beta_{i}+\gamma_{i}^{2}\right) z_{i}(t) z_{i}^{\prime}(t) e^{2 \lambda t}+\sum_{i=1}^{n} \alpha_{i}\left(\alpha_{i} z_{i}^{\prime}(t)+\gamma_{i} z_{i}(t)\right) e^{2 \lambda t} \\
& \times\left[-a_{i}(t) z_{i}^{\prime}(t)-b_{i}(t) z_{i}(t)+\sum_{j=1}^{n} c_{i j}(t) \widetilde{A}_{j}\left(z_{j}(t)\right)+\sum_{j=1}^{n} d_{i j}(t) \widetilde{B}_{j}\left(z_{j}\left(t-q_{i j}(t)\right)\right)\right. \\
& +\sum_{j=1}^{n} \sum_{l=1}^{n} \theta_{i j l}(t)\left(Q_{j}\left(y_{j}\left(t-\eta_{i j l}(t)\right)\right) Q_{l}\left(y_{l}\left(t-\xi_{i j l}(t)\right)\right)\right. \\
& -Q_{j}\left(y_{j}\left(t-\eta_{i j l}(t)\right)\right) Q_{l}\left(x_{l}\left(t-\xi_{i j l}(t)\right)\right)+Q_{j}\left(y_{j}\left(t-\eta_{i j l}(t)\right)\right) Q_{l}\left(x_{l}\left(t-\xi_{i j l}(t)\right)\right)
\end{aligned}
$$




$$
\begin{aligned}
& \left.-Q_{j}\left(x_{j}\left(t-\eta_{i j l}(t)\right)\right) Q_{l}\left(x_{l}\left(t-\xi_{i j l}(t)\right)\right)\right) \\
& +\sum_{j=1}^{n} h_{i j}(t) \int_{0}^{+\infty} \sigma_{i j}(u) \widetilde{K}_{j}\left(z_{j}(t-u)\right) d u+\sum_{j=1}^{n} \sum_{l=1}^{n} p_{i j l}(t) \\
& \times\left(\int_{0}^{+\infty} \hat{\sigma}_{i j l}(u) R_{j}\left(y_{j}(t-u)\right) d u \int_{0}^{+\infty} \bar{\sigma}_{i j l}(u) R_{l}\left(y_{l}(t-u)\right) d u\right. \\
& -\int_{0}^{+\infty} \hat{\sigma}_{i j l}(u) R_{j}\left(y_{j}(t-u)\right) d u \int_{0}^{+\infty} \bar{\sigma}_{i j l}(u) R_{l}\left(x_{l}(t-u)\right) d u \\
& +\int_{0}^{+\infty} \hat{\sigma}_{i j l}(u) R_{j}\left(y_{j}(t-u)\right) d u \int_{0}^{+\infty} \bar{\sigma}_{i j l}(u) R_{l}\left(x_{l}(t-u)\right) d u \\
& \left.\left.-\int_{0}^{+\infty} \hat{\sigma}_{i j l}(u) R_{j}\left(x_{j}(t-u)\right) d u \int_{0}^{+\infty} \bar{\sigma}_{i j l}(u) R_{l}\left(x_{l}(t-u)\right) d u\right)\right] \\
& +\sum_{i=1}^{n} \alpha_{i} \gamma_{i}\left(z_{i}^{\prime}(t)\right)^{2} e^{2 \lambda t}+\frac{1}{2} \sum_{i=1}^{n} \sum_{j=1}^{n}\left(\alpha_{i}^{2} d_{i j}^{+}+\left|\alpha_{i} \gamma_{i}\right| d_{i j}^{+}\right) e^{2 \lambda q_{i j}^{+}} L_{j}^{B} \\
& \times\left[z_{j}^{2}(t) \frac{1}{1-\dot{q}_{i j}^{+}} e^{2 \lambda t}-z_{j}^{2}\left(t-q_{i j}(t)\right) e^{2 \lambda\left(t-q_{i j}(t)\right)} \frac{1-q_{i j}^{\prime}(t)}{1-\dot{q}_{i j}^{+}}\right] \\
& +\frac{1}{2} \sum_{i=1}^{n} \sum_{l=1}^{n} \sum_{j=1}^{n}\left(\alpha_{l}^{2}+\left|\alpha_{l} \gamma_{l}\right|\right) \theta_{l j i}^{+} M_{j}^{Q} L_{i}^{Q} e^{2 \lambda \xi_{l j i}^{+}} \\
& \times\left[z_{i}^{2}(t) \frac{1}{1-\dot{\xi}_{l j i}^{+}} e^{2 \lambda t}-z_{i}^{2}\left(t-\xi_{l j i}(t)\right) e^{2 \lambda\left(t-\xi_{l j i}(t)\right)} \frac{1-\xi_{l j i}^{\prime}(t)}{1-\dot{\xi}_{l j i}^{+}}\right] \\
& +\frac{1}{2} \sum_{i=1}^{n} \sum_{j=1}^{n} \sum_{l=1}^{n}\left(\alpha_{j}^{2}+\left|\alpha_{j} \gamma_{j}\right|\right) \theta_{j i l}^{+} L_{i}^{Q} M_{l}^{Q} e^{2 \lambda \eta_{j i l}^{+}} \\
& \times\left[z_{i}^{2}(t) \frac{1}{1-\dot{\eta}_{j i l}^{+}} e^{2 \lambda t}-z_{i}^{2}\left(t-\eta_{j i l}(t)\right) e^{2 \lambda\left(t-\eta_{j i l}(t)\right)} \frac{1-\eta_{j i l}^{\prime}(t)}{1-\dot{\eta}_{j i l}^{+}}\right] \\
& +\frac{1}{2} \sum_{i=1}^{n} \sum_{j=1}^{n}\left(\alpha_{i}^{2} h_{i j}^{+}+\left|\alpha_{i} \gamma_{i}\right| h_{i j}^{+}\right) L_{j}^{K} \\
& \times\left[\int_{0}^{+\infty}\left|\sigma_{i j}(u)\right| e^{2 \lambda u} d u z_{j}^{2}(t) e^{2 \lambda t}-\int_{0}^{+\infty}\left|\sigma_{i j}(u)\right| z_{j}^{2}(t-u) d u e^{2 \lambda t}\right] \\
& +\frac{1}{2} \sum_{i=1}^{n} \sum_{l=1}^{n} \sum_{j=1}^{n}\left(\alpha_{l}^{2}+\left|\alpha_{l} \gamma_{l}\right|\right) p_{l j i}^{+} M_{j}^{R} L_{i}^{R} \int_{0}^{+\infty}\left|\hat{\sigma}_{l j i}(u)\right| d u \\
& \times\left[\int_{0}^{+\infty}\left|\bar{\sigma}_{l j i}(u)\right| e^{2 \lambda u} d u z_{i}^{2}(t) e^{2 \lambda t}-\int_{0}^{+\infty}\left|\bar{\sigma}_{l j i}(u)\right| z_{i}^{2}(t-u) d u e^{2 \lambda t}\right] \\
& +\frac{1}{2} \sum_{i=1}^{n} \sum_{j=1}^{n} \sum_{l=1}^{n}\left(\alpha_{j}^{2}+\left|\alpha_{j} \gamma_{j}\right|\right) p_{j i l}^{+} M_{l}^{R} L_{i}^{R} \int_{0}^{+\infty}\left|\bar{\sigma}_{j i l}(u)\right| d u \\
& \times\left[\int_{0}^{+\infty}\left|\hat{\sigma}_{j i l}(u)\right| e^{2 \lambda u} d u z_{i}^{2}(t) e^{2 \lambda t}-\int_{0}^{+\infty}\left|\hat{\sigma}_{j i l}(u)\right| z_{i}^{2}(t-u) d u e^{2 \lambda t}\right] \\
& \leq e^{2 \lambda t}\left\{\sum_{i=1}^{n}\left(\beta_{i}+\gamma_{i}^{2}+2 \lambda \alpha_{i} \gamma_{i}-a_{i}(t) \alpha_{i} \gamma_{i}-b_{i}(t) \alpha_{i}^{2}\right) z_{i}(t) z_{i}^{\prime}(t)\right.
\end{aligned}
$$




$$
\begin{aligned}
& +\sum_{i=1}^{n}\left(\lambda \alpha_{i}^{2}+\alpha_{i} \gamma_{i}-a_{i}(t) \alpha_{i}^{2}\right)\left(z_{i}^{\prime}(t)\right)^{2}-\sum_{i=1}^{n}\left(b_{i}(t) \alpha_{i} \gamma_{i}-\lambda \beta_{i}-\lambda \gamma_{i}^{2}\right) z_{i}^{2}(t) \\
& +\frac{1}{2} \sum_{i=1}^{n} \sum_{j=1}^{n}\left(\alpha_{i}^{2} d_{i j}^{+}+\left|\alpha_{i} \gamma_{i}\right| d_{i j}^{+}\right) L_{j}^{B} e^{2 \lambda q_{i j}^{+}} z_{j}^{2}(t) \frac{1}{1-\dot{q}_{i j}^{+}} \\
& +\frac{1}{2} \sum_{i=1}^{n} \sum_{l=1}^{n} \sum_{j=1}^{n}\left(\alpha_{l}^{2}+\left|\alpha_{l} \gamma_{l}\right|\right) \theta_{l j i}^{+} M_{j}^{Q} L_{i}^{Q} e^{2 \lambda \xi_{l j i}^{+}} z_{i}^{2}(t) \frac{1}{1-\dot{\xi}_{l j i}^{+}} \\
& +\frac{1}{2} \sum_{i=1}^{n} \sum_{j=1}^{n} \sum_{l=1}^{n}\left(\alpha_{j}^{2}+\left|\alpha_{j} \gamma_{j}\right|\right) \theta_{j i l}^{+} L_{i}^{Q} M_{l}^{Q} e^{2 \lambda \eta_{j i l}^{+}} z_{i}^{2}(t) \frac{1}{1-\dot{\eta}_{j i l}^{+}} \\
& -\frac{1}{2} \sum_{i=1}^{n} \sum_{j=1}^{n}\left(\alpha_{i}^{2} d_{i j}^{+}+\left|\alpha_{i} \gamma_{i}\right| d_{i j}^{+}\right) L_{j}^{B} z_{j}^{2}\left(t-q_{i j}(t)\right) \\
& -\frac{1}{2} \sum_{i=1}^{n} \sum_{l=1}^{n} \sum_{j=1}^{n}\left(\alpha_{l}^{2}+\left|\alpha_{l} \gamma_{l}\right|\right) \theta_{l j i}^{+} M_{j}^{Q} L_{i}^{Q} z_{i}^{2}\left(t-\xi_{l j i}(t)\right) \\
& -\frac{1}{2} \sum_{i=1}^{n} \sum_{j=1}^{n} \sum_{l=1}^{n}\left(\alpha_{j}^{2}+\left|\alpha_{j} \gamma_{j}\right|\right) \theta_{j i l}^{+} L_{i}^{Q} M_{l}^{Q} z_{i}^{2}\left(t-\eta_{j i l}(t)\right) \\
& +\sum_{i=1}^{n} \sum_{j=1}^{n}\left(\alpha_{i}^{2}\left|z_{i}^{\prime}(t)\right|+\left|\alpha_{i} \gamma_{i}\right|\left|z_{i}(t)\right|\right)\left|c_{i j}(t)\right|\left|\widetilde{A}_{j}\left(z_{j}(t)\right)\right| \\
& +\sum_{i=1}^{n} \sum_{j=1}^{n}\left(\alpha_{i}^{2}\left|z_{i}^{\prime}(t)\right|+\left|\alpha_{i} \gamma_{i}\right|\left|z_{i}(t)\right|\right)\left|d_{i j}(t)\right|\left|\widetilde{B}_{j}\left(z_{j}\left(t-q_{i j}(t)\right)\right)\right| \\
& +\sum_{i=1}^{n}\left(\alpha_{i}^{2}\left|z_{i}^{\prime}(t)\right|+\left|\alpha_{i} \gamma_{i}\right|\left|z_{i}(t)\right|\right) \\
& \times \sum_{j=1}^{n} \sum_{l=1}^{n}\left|\theta_{i j l}(t)\right|\left(M_{j}^{Q} L_{l}^{Q}\left|z_{l}\left(t-\xi_{i j l}(t)\right)\right|+M_{l}^{Q} L_{j}^{Q}\left|z_{j}\left(t-\eta_{i j l}(t)\right)\right|\right) \\
& +\sum_{i=1}^{n} \sum_{j=1}^{n}\left(\alpha_{i}^{2}\left|z_{i}^{\prime}(t)\right|+\left|\alpha_{i} \gamma_{i}\right|\left|z_{i}(t)\right|\right)\left|h_{i j}(t)\right| \int_{0}^{+\infty}\left|\sigma_{i j}(u)\right|\left|\widetilde{K}_{j}\left(z_{j}(t-u)\right)\right| d u \\
& +\sum_{i=1}^{n} \sum_{j=1}^{n} \sum_{l=1}^{n}\left(\alpha_{i}^{2}\left|z_{i}^{\prime}(t)\right|+\left|\alpha_{i} \gamma_{i}\right|\left|z_{i}(t)\right|\right)\left|p_{i j l}(t)\right| \int_{0}^{+\infty}\left|\hat{\sigma}_{i j l}(u)\right| d u \\
& \times \int_{0}^{+\infty}\left|\bar{\sigma}_{i j l}(u)\right|\left|z_{l}(t-u)\right| d u M_{j}^{R} L_{l}^{R} \\
& +\sum_{i=1}^{n} \sum_{j=1}^{n} \sum_{l=1}^{n}\left(\alpha_{i}^{2}\left|z_{i}^{\prime}(t)\right|+\left|\alpha_{i} \gamma_{i}\right|\left|z_{i}(t)\right|\right)\left|p_{i j l}(t)\right| \int_{0}^{+\infty}\left|\bar{\sigma}_{i j l}(u)\right| d u \\
& \times \int_{0}^{+\infty}\left|\hat{\sigma}_{i j l}(u)\right|\left|z_{j}(t-u)\right| d u M_{l}^{R} L_{j}^{R} \\
& +\frac{1}{2} \sum_{i=1}^{n} \sum_{j=1}^{n}\left(\alpha_{i}^{2} h_{i j}^{+}+\left|\alpha_{i} \gamma_{i}\right| h_{i j}^{+}\right) L_{j}^{K} \int_{0}^{+\infty}\left|\sigma_{i j}(u)\right| e^{2 \lambda u} d u z_{j}^{2}(t) \\
& -\frac{1}{2} \sum_{i=1}^{n} \sum_{j=1}^{n}\left(\alpha_{i}^{2} h_{i j}^{+}+\left|\alpha_{i} \gamma_{i}\right| h_{i j}^{+}\right) L_{j}^{K} \int_{0}^{+\infty}\left|\sigma_{i j}(u)\right| z_{j}^{2}(t-u) d u
\end{aligned}
$$




$$
\begin{aligned}
& +\frac{1}{2} \sum_{i=1}^{n} \sum_{l=1}^{n} \sum_{j=1}^{n}\left(\alpha_{l}^{2}+\left|\alpha_{l} \gamma_{l}\right|\right) p_{l j i}^{+} \int_{0}^{+\infty}\left|\hat{\sigma}_{l j i}(u)\right| d u \int_{0}^{+\infty}\left|\bar{\sigma}_{l j i}(u)\right| e^{2 \lambda u} d u z_{i}^{2}(t) M_{j}^{R} L_{i}^{R} \\
& -\frac{1}{2} \sum_{i=1}^{n} \sum_{l=1}^{n} \sum_{j=1}^{n}\left(\alpha_{l}^{2}+\left|\alpha_{l} \gamma_{l}\right|\right) p_{l j i}^{+} \int_{0}^{+\infty}\left|\hat{\sigma}_{l j i}(u)\right| d u \int_{0}^{+\infty}\left|\bar{\sigma}_{l j i}(u)\right| z_{i}^{2}(t-u) d u M_{j}^{R} L_{i}^{R} \\
& +\frac{1}{2} \sum_{i=1}^{n} \sum_{j=1}^{n} \sum_{l=1}^{n}\left(\alpha_{j}^{2}+\left|\alpha_{j} \gamma_{j}\right|\right) p_{j i l}^{+} \int_{0}^{+\infty}\left|\bar{\sigma}_{j i l}(u)\right| d u \int_{0}^{+\infty}\left|\hat{\sigma}_{j i l}(u)\right| e^{2 \lambda u} d u z_{i}^{2}(t) M_{l}^{R} L_{i}^{R} \\
& \left.-\frac{1}{2} \sum_{i=1}^{n} \sum_{j=1}^{n} \sum_{l=1}^{n}\left(\alpha_{j}^{2}+\left|\alpha_{j} \gamma_{j}\right|\right) p_{j i l}^{+} \int_{0}^{+\infty}\left|\bar{\sigma}_{j i l}(u)\right| d u \int_{0}^{+\infty}\left|\hat{\sigma}_{j i l}(u)\right| z_{i}^{2}(t-u) d u M_{l}^{R} L_{i}^{R}\right\} \\
& =e^{2 \lambda t}\left\{\sum_{i=1}^{n}\left(\beta_{i}+\gamma_{i}^{2}+2 \lambda \alpha_{i} \gamma_{i}-a_{i}(t) \alpha_{i} \gamma_{i}-b_{i}(t) \alpha_{i}^{2}\right) z_{i}(t) z_{i}^{\prime}(t)\right. \\
& +\sum_{i=1}^{n}\left(\lambda \alpha_{i}^{2}+\alpha_{i} \gamma_{i}-a_{i}(t) \alpha_{i}^{2}\right)\left(z_{i}^{\prime}(t)\right)^{2} \\
& +\sum_{i=1}^{n}\left[-b_{i}(t) \alpha_{i} \gamma_{i}+\lambda \beta_{i}+\lambda \gamma_{i}^{2}+\frac{1}{2} \sum_{j=1}^{n}\left(\alpha_{j}^{2} d_{j i}^{+}+\left|\alpha_{j} \gamma_{j}\right| d_{j i}^{+}\right) L_{i}^{B} e^{2 \lambda q_{j i}^{+}} \frac{1}{1-\dot{q}_{j i}^{+}}\right. \\
& +\frac{1}{2} \sum_{l=1}^{n} \sum_{j=1}^{n}\left(\alpha_{l}^{2}+\left|\alpha_{l} \gamma_{l}\right|\right) \theta_{l j i}^{+} M_{j}^{Q} L_{i}^{Q} e^{2 \lambda \xi_{l j i}^{+}} \frac{1}{1-\dot{\xi}_{l j i}^{+}} \\
& +\frac{1}{2} \sum_{j=1}^{n} \sum_{l=1}^{n}\left(\alpha_{j}^{2}+\left|\alpha_{j} \gamma_{j}\right|\right) \theta_{j i l}^{+} L_{i}^{Q} M_{l}^{Q} e^{2 \lambda \eta_{j i l}^{+}} \frac{1}{1-\dot{\eta}_{j i l}^{+}} \\
& +\frac{1}{2} \sum_{j=1}^{n}\left(\alpha_{j}^{2} h_{j i}^{+}+\left|\alpha_{j} \gamma_{j}\right| h_{j i}^{+}\right) L_{i}^{K} \int_{0}^{+\infty}\left|\sigma_{j i}(u)\right| e^{2 \lambda u} d u \\
& +\frac{1}{2} \sum_{l=1}^{n} \sum_{j=1}^{n}\left(\alpha_{l}^{2}+\left|\alpha_{l} \gamma_{l}\right|\right) p_{l j i}^{+} \int_{0}^{+\infty}\left|\hat{\sigma}_{l j i}(u)\right| d u \int_{0}^{+\infty}\left|\bar{\sigma}_{l j i}(u)\right| e^{2 \lambda u} d u M_{j}^{R} L_{i}^{R} \\
& \left.+\frac{1}{2} \sum_{j=1}^{n} \sum_{l=1}^{n}\left(\alpha_{j}^{2}+\left|\alpha_{j} \gamma_{j}\right|\right) p_{j i l}^{+} \int_{0}^{+\infty}\left|\bar{\sigma}_{j i l}(u)\right| d u \int_{0}^{+\infty}\left|\hat{\sigma}_{j i l}(u)\right| e^{2 \lambda u} d u M_{l}^{R} L_{i}^{R}\right] z_{i}^{2}(t) \\
& -\frac{1}{2} \sum_{i=1}^{n} \sum_{j=1}^{n}\left(\alpha_{i}^{2} d_{i j}^{+}+\left|\alpha_{i} \gamma_{i}\right| d_{i j}^{+}\right) L_{j}^{B} z_{j}^{2}\left(t-q_{i j}(t)\right) \\
& -\frac{1}{2} \sum_{i=1}^{n} \sum_{l=1}^{n} \sum_{j=1}^{n}\left(\alpha_{l}^{2}+\left|\alpha_{l} \gamma_{l}\right|\right) \theta_{l j i}^{+} M_{j}^{Q} L_{i}^{Q} z_{i}^{2}\left(t-\xi_{l j i}(t)\right) \\
& -\frac{1}{2} \sum_{i=1}^{n} \sum_{j=1}^{n} \sum_{l=1}^{n}\left(\alpha_{j}^{2}+\left|\alpha_{j} \gamma_{j}\right|\right) \theta_{j i l}^{+} L_{i}^{Q} M_{l}^{Q} z_{i}^{2}\left(t-\eta_{j i l}(t)\right) \\
& -\frac{1}{2} \sum_{i=1}^{n} \sum_{j=1}^{n}\left(\alpha_{i}^{2} h_{i j}^{+}+\left|\alpha_{i} \gamma_{i}\right| h_{i j}^{+}\right) L_{j}^{K} \int_{0}^{+\infty}\left|\sigma_{i j}(u)\right| z_{j}^{2}(t-u) d u \\
& -\frac{1}{2} \sum_{i=1}^{n} \sum_{l=1}^{n} \sum_{j=1}^{n}\left(\alpha_{l}^{2}+\left|\alpha_{l} \gamma_{l}\right|\right) p_{l j i}^{+} \int_{0}^{+\infty}\left|\hat{\sigma}_{l j i}(u)\right| d u \int_{0}^{+\infty}\left|\bar{\sigma}_{l j i}(u)\right| z_{i}^{2}(t-u) d u M_{j}^{R} L_{i}^{R}
\end{aligned}
$$




$$
\begin{aligned}
& -\frac{1}{2} \sum_{i=1}^{n} \sum_{j=1}^{n} \sum_{l=1}^{n}\left(\alpha_{j}^{2}+\left|\alpha_{j} \gamma_{j}\right|\right) p_{j i l}^{+} \int_{0}^{+\infty}\left|\bar{\sigma}_{j i l}(u)\right| d u \int_{0}^{+\infty}\left|\hat{\sigma}_{j i l}(u)\right| z_{i}^{2}(t-u) d u M_{l}^{R} L_{i}^{R} \\
& +\sum_{i=1}^{n} \sum_{j=1}^{n}\left(\alpha_{i}^{2}\left|z_{i}^{\prime}(t)\right|+\left|\alpha_{i} \gamma_{i}\right|\left|z_{i}(t)\right|\right)\left|c_{i j}(t)\right|\left|\widetilde{A}_{j}\left(z_{j}(t)\right)\right| \\
& +\sum_{i=1}^{n} \sum_{j=1}^{n}\left(\alpha_{i}^{2}\left|z_{i}^{\prime}(t)\right|+\left|\alpha_{i} \gamma_{i}\right|\left|z_{i}(t)\right|\right)\left|d_{i j}(t)\right|\left|\widetilde{B}_{j}\left(z_{j}\left(t-q_{i j}(t)\right)\right)\right| \\
& +\sum_{i=1}^{n}\left(\alpha_{i}^{2}\left|z_{i}^{\prime}(t)\right|+\left|\alpha_{i} \gamma_{i}\right|\left|z_{i}(t)\right|\right) \\
& \times \sum_{j=1}^{n} \sum_{l=1}^{n}\left|\theta_{i j l}(t)\right|\left(M_{j}^{Q} L_{l}^{Q}\left|z_{l}\left(t-\xi_{i j l}(t)\right)\right|+M_{l}^{Q} L_{j}^{Q}\left|z_{j}\left(t-\eta_{i j l}(t)\right)\right|\right) \\
& +\sum_{i=1}^{n} \sum_{j=1}^{n}\left(\alpha_{i}^{2}\left|z_{i}^{\prime}(t)\right|+\left|\alpha_{i} \gamma_{i}\right|\left|z_{i}(t)\right|\right)\left|h_{i j}(t)\right| \int_{0}^{+\infty}\left|\sigma_{i j}(u)\right|\left|\widetilde{K}_{j}\left(z_{j}(t-u)\right)\right| d u \\
& +\sum_{i=1}^{n} \sum_{j=1}^{n} \sum_{l=1}^{n}\left(\alpha_{i}^{2}\left|z_{i}^{\prime}(t)\right|+\left|\alpha_{i} \gamma_{i}\right|\left|z_{i}(t)\right|\right)\left|p_{i j l}(t)\right| \\
& \times \int_{0}^{+\infty}\left|\hat{\sigma}_{i j l}(u)\right| d u \int_{0}^{+\infty}\left|\bar{\sigma}_{i j l}(u)\right|\left|z_{l}(t-u)\right| d u M_{j}^{R} L_{l}^{R} \\
& +\sum_{i=1}^{n} \sum_{j=1}^{n} \sum_{l=1}^{n}\left(\alpha_{i}^{2}\left|z_{i}^{\prime}(t)\right|+\left|\alpha_{i} \gamma_{i}\right|\left|z_{i}(t)\right|\right)\left|p_{i j l}(t)\right| \\
& \left.\times \int_{0}^{+\infty}\left|\bar{\sigma}_{i j l}(u)\right| d u \int_{0}^{+\infty}\left|\hat{\sigma}_{i j l}(u)\right|\left|z_{j}(t-u)\right| d u M_{l}^{R} L_{j}^{R}\right\}, \quad \forall t \in[0,+\infty) .
\end{aligned}
$$

It follows from $\left(G_{1}\right)$ and $P Q \leq \frac{1}{2}\left(P^{2}+Q^{2}\right)(P, Q \in \mathbb{R})$ that

$$
\begin{aligned}
& \sum_{i=1}^{n} \sum_{j=1}^{n}\left(\alpha_{i}^{2}\left|z_{i}^{\prime}(t)\right|+\left|\alpha_{i} \gamma_{i}\right|\left|z_{i}(t)\right|\right)\left|c_{i j}(t)\right|\left|\widetilde{A}_{j}\left(z_{j}(t)\right)\right| \\
& \leq \leq \frac{1}{2} \sum_{i=1}^{n} \sum_{j=1}^{n} \alpha_{i}^{2}\left|c_{i j}(t)\right| L_{j}^{A}\left(\left(z_{i}^{\prime}(t)\right)^{2}+z_{j}^{2}(t)\right) \\
& \quad+\frac{1}{2} \sum_{i=1}^{n} \sum_{j=1}^{n}\left|\alpha_{i} \gamma_{i}\right|\left|c_{i j}(t)\right| L_{j}^{A}\left(z_{i}^{2}(t)+z_{j}^{2}(t)\right) \\
& =\frac{1}{2} \sum_{i=1}^{n} \sum_{j=1}^{n} \alpha_{i}^{2}\left|c_{i j}(t)\right| L_{j}^{A}\left(z_{i}^{\prime}(t)\right)^{2} \\
& \quad+\frac{1}{2} \sum_{i=1}^{n} \sum_{j=1}^{n}\left(\left|\alpha_{i} \gamma_{i}\right|\left|c_{i j}(t)\right| L_{j}^{A}+\alpha_{j}^{2}\left|c_{j i}(t)\right| L_{i}^{A}+\left|\alpha_{j} \gamma_{j}\right|\left|c_{j i}(t)\right| L_{i}^{A}\right) z_{i}^{2}(t), \\
& \sum_{i=1}^{n} \sum_{j=1}^{n}\left(\alpha_{i}^{2}\left|z_{i}^{\prime}(t)\right|+\left|\alpha_{i} \gamma_{i}\right|\left|z_{i}(t)\right|\right)\left|d_{i j}(t)\right|\left|\widetilde{B}_{j}\left(z_{j}\left(t-q_{i j}(t)\right)\right)\right| \\
& \leq \\
& \frac{1}{2} \sum_{i=1}^{n} \sum_{j=1}^{n} \alpha_{i}^{2}\left|d_{i j}(t)\right| L_{j}^{B}\left(\left(z_{i}^{\prime}(t)\right)^{2}+z_{j}^{2}\left(t-q_{i j}(t)\right)\right)
\end{aligned}
$$




$$
\begin{aligned}
& +\frac{1}{2} \sum_{i=1}^{n} \sum_{j=1}^{n}\left|\alpha_{i} \gamma_{i}\right|\left|d_{i j}(t)\right| L_{j}^{B}\left(z_{i}^{2}(t)+z_{j}^{2}\left(t-q_{i j}(t)\right)\right) \\
& =\frac{1}{2} \sum_{i=1}^{n} \sum_{j=1}^{n} \alpha_{i}^{2}\left|d_{i j}(t)\right| L_{j}^{B}\left(z_{i}^{\prime}(t)\right)^{2}+\frac{1}{2} \sum_{i=1}^{n} \sum_{j=1}^{n}\left|\alpha_{i} \gamma_{i}\right|\left|d_{i j}(t)\right| L_{j}^{B} z_{i}^{2}(t) \\
& +\frac{1}{2} \sum_{i=1}^{n} \sum_{j=1}^{n}\left(\alpha_{i}^{2}\left|d_{i j}(t)\right| L_{j}^{B}+\left|\alpha_{i} \gamma_{i}\right|\left|d_{i j}(t)\right| L_{j}^{B}\right) z_{j}^{2}\left(t-q_{i j}(t)\right), \\
& \sum_{i=1}^{n}\left(\alpha_{i}^{2}\left|z_{i}^{\prime}(t)\right|+\left|\alpha_{i} \gamma_{i}\right|\left|z_{i}(t)\right|\right) \\
& \times \sum_{j=1}^{n} \sum_{l=1}^{n}\left|\theta_{i j l}(t)\right|\left(M_{j}^{Q} L_{l}^{Q}\left|z_{l}\left(t-\xi_{i j l}(t)\right)\right|+L_{j}^{Q}\left|z_{j}\left(t-\eta_{i j l}(t)\right)\right| M_{l}^{Q}\right) \\
& \leq \frac{1}{2} \sum_{i=1}^{n} \sum_{j=1}^{n} \sum_{l=1}^{n} \alpha_{i}^{2}\left|\theta_{i j l}(t)\right| M_{j}^{Q} L_{l}^{Q}\left(\left(z_{i}^{\prime}(t)\right)^{2}+z_{l}^{2}\left(t-\xi_{i j l}(t)\right)\right) \\
& +\frac{1}{2} \sum_{i=1}^{n} \sum_{j=1}^{n} \sum_{l=1}^{n}\left|\alpha_{i} \gamma_{i}\right|\left|\theta_{i j l}(t)\right| M_{j}^{Q} L_{l}^{Q}\left(\left(z_{i}(t)\right)^{2}+z_{l}^{2}\left(t-\xi_{i j l}(t)\right)\right) \\
& +\frac{1}{2} \sum_{i=1}^{n} \sum_{j=1}^{n} \sum_{l=1}^{n} \alpha_{i}^{2}\left|\theta_{i j l}(t)\right| L_{j}^{Q} M_{l}^{Q}\left(\left(z_{i}^{\prime}(t)\right)^{2}+z_{j}^{2}\left(t-\eta_{i j l}(t)\right)\right) \\
& +\frac{1}{2} \sum_{i=1}^{n} \sum_{j=1}^{n} \sum_{l=1}^{n}\left|\alpha_{i} \gamma_{i}\right|\left|\theta_{i j l}(t)\right| L_{j}^{Q} M_{l}^{Q}\left(\left(z_{i}(t)\right)^{2}+z_{j}^{2}\left(t-\eta_{i j l}(t)\right)\right) \\
& =\frac{1}{2} \sum_{i=1}^{n} \sum_{j=1}^{n} \sum_{l=1}^{n} \alpha_{i}^{2}\left|\theta_{i j l}(t)\right|\left(M_{j}^{Q} L_{l}^{Q}+L_{j}^{Q} M_{l}^{Q}\right)\left(z_{i}^{\prime}(t)\right)^{2} \\
& +\frac{1}{2} \sum_{i=1}^{n} \sum_{j=1}^{n} \sum_{l=1}^{n}\left|\alpha_{i} \gamma_{i}\right|\left|\theta_{i j l}(t)\right|\left(M_{j}^{Q} L_{l}^{Q}+L_{j}^{Q} M_{l}^{Q}\right)\left(z_{i}(t)\right)^{2} \\
& +\frac{1}{2} \sum_{i=1}^{n} \sum_{j=1}^{n} \sum_{l=1}^{n}\left(\alpha_{i}^{2}+\left|\alpha_{i} \gamma_{i}\right|\right)\left|\theta_{i j l}(t)\right| M_{j}^{Q} L_{l}^{Q} z_{l}^{2}\left(t-\xi_{i j l}(t)\right) \\
& +\frac{1}{2} \sum_{i=1}^{n} \sum_{j=1}^{n} \sum_{l=1}^{n}\left(\alpha_{i}^{2}+\left|\alpha_{i} \gamma_{i}\right|\right)\left|\theta_{i j l}(t)\right| L_{j}^{Q} M_{l}^{Q} z_{j}^{2}\left(t-\eta_{i j l}(t)\right) \\
& =\frac{1}{2} \sum_{i=1}^{n} \sum_{j=1}^{n} \sum_{l=1}^{n} \alpha_{i}^{2}\left|\theta_{i j l}(t)\right|\left(M_{j}^{Q} L_{l}^{Q}+L_{j}^{Q} M_{l}^{Q}\right)\left(z_{i}^{\prime}(t)\right)^{2} \\
& +\frac{1}{2} \sum_{i=1}^{n} \sum_{j=1}^{n} \sum_{l=1}^{n}\left|\alpha_{i} \gamma_{i}\right|\left|\theta_{i j l}(t)\right|\left(M_{j}^{Q} L_{l}^{Q}+L_{j}^{Q} M_{l}^{Q}\right)\left(z_{i}(t)\right)^{2} \\
& +\frac{1}{2} \sum_{l=1}^{n} \sum_{j=1}^{n} \sum_{i=1}^{n}\left(\alpha_{l}^{2}+\left|\alpha_{l} \gamma_{l}\right|\right)\left|\theta_{l j i}(t)\right| M_{j}^{Q} L_{i}^{Q} z_{i}^{2}\left(t-\xi_{l j i}(t)\right) \\
& +\frac{1}{2} \sum_{j=1}^{n} \sum_{i=1}^{n} \sum_{l=1}^{n}\left(\alpha_{j}^{2}+\left|\alpha_{j} \gamma_{j}\right|\right)\left|\theta_{j i l}(t)\right| L_{i}^{Q} M_{l}^{Q} z_{i}^{2}\left(t-\eta_{j i l}(t)\right),
\end{aligned}
$$




$$
\begin{aligned}
& \sum_{i=1}^{n} \sum_{j=1}^{n}\left(\alpha_{i}^{2}\left|z_{i}^{\prime}(t)\right|+\left|\alpha_{i} \gamma_{i}\right|\left|z_{i}(t)\right|\right)\left|h_{i j}(t)\right| \int_{0}^{+\infty}\left|\sigma_{i j}(u)\right|\left|\widetilde{K}_{j}\left(z_{j}(t-u)\right)\right| d u \\
& \leq \frac{1}{2} \sum_{i=1}^{n} \sum_{j=1}^{n} \alpha_{i}^{2}\left|h_{i j}(t)\right| L_{j}^{K} \int_{0}^{+\infty}\left|\sigma_{i j}(u)\right| d u\left(z_{i}^{\prime}(t)\right)^{2} \\
&+\frac{1}{2} \sum_{i=1}^{n} \sum_{j=1}^{n}\left|\alpha_{i} \gamma_{i}\right|\left|h_{i j}(t)\right| L_{j}^{K} \int_{0}^{+\infty}\left|\sigma_{i j}(u)\right| d u z_{i}^{2}(t) \\
&+\frac{1}{2} \sum_{i=1}^{n} \sum_{j=1}^{n}\left(\alpha_{i}^{2}\left|h_{i j}(t)\right| L_{j}^{K}+\left|\alpha_{i} \gamma_{i}\right|\left|h_{i j}(t)\right| L_{j}^{K}\right) \int_{0}^{+\infty}\left|\sigma_{i j}(u)\right| z_{j}^{2}(t-u) d u, \\
& \sum_{i=1}^{n} \sum_{j=1}^{n} \sum_{l=1}^{n}\left(\alpha_{i}^{2}\left|z_{i}^{\prime}(t)\right|+\left|\alpha_{i} \gamma_{i}\right|\left|z_{i}(t)\right|\right)\left|p_{i j l}(t)\right| \\
& \quad \times \int_{0}^{+\infty}\left|\hat{\sigma}_{i j l}(u)\right| d u \int_{0}^{+\infty}\left|\bar{\sigma}_{i j l}(u)\right|\left|z_{l}(t-u)\right| d u M_{j}^{R} L_{l}^{R} \\
& \leq \frac{1}{2} \sum_{i=1}^{n} \sum_{j=1}^{n} \sum_{l=1}^{n} \alpha_{i}^{2}\left|p_{i j l}(t)\right| M_{j}^{R} L_{l}^{R} \int_{0}^{+\infty}\left|\hat{\sigma}_{i j l}(u)\right| d u \int_{0}^{+\infty}\left|\bar{\sigma}_{i j l}(u)\right| d u\left(z_{i}^{\prime}(t)\right)^{2} \\
&+\frac{1}{2} \sum_{i=1}^{n} \sum_{j=1}^{n} \sum_{l=1}^{n}\left|\alpha_{i} \gamma_{i}\right|\left|p_{i j l}(t)\right| M_{j}^{R} L_{l}^{R} \int_{0}^{+\infty}\left|\hat{\sigma}_{i j l}(u)\right| d u \int_{0}^{+\infty}\left|\bar{\sigma}_{i j l}(u)\right| d u z_{i}^{2}(t) \\
&+\frac{1}{2} \sum_{l=1}^{n} \sum_{j=1}^{n} \sum_{i=1}^{n}\left(\alpha_{l}^{2}+\left|\alpha_{l} \gamma_{l}\right|\right)\left|p_{l j i}(t)\right| M_{j}^{R} L_{i}^{R} \int_{0}^{+\infty}\left|\hat{\sigma}_{l j i}(u)\right| d u \\
& \times \int_{0}^{+\infty}\left|\bar{\sigma}_{l j i}(u)\right| z_{i}^{2}(t-u) d u,
\end{aligned}
$$

and

$$
\begin{aligned}
\sum_{i=1}^{n} & \sum_{j=1}^{n} \sum_{l=1}^{n}\left(\alpha_{i}^{2}\left|z_{i}^{\prime}(t)\right|+\left|\alpha_{i} \gamma_{i}\right|\left|z_{i}(t)\right|\right)\left|p_{i j l}(t)\right| \\
& \times \int_{0}^{+\infty}\left|\bar{\sigma}_{i j l}(u)\right| d u \int_{0}^{+\infty}\left|\hat{\sigma}_{i j l}(u)\right|\left|z_{j}(t-u)\right| d u M_{l}^{R} L_{j}^{R} \\
\leq & \frac{1}{2} \sum_{i=1}^{n} \sum_{j=1}^{n} \sum_{l=1}^{n} \alpha_{i}^{2}\left|p_{i j l}(t)\right| M_{l}^{R} L_{j}^{R} \int_{0}^{+\infty}\left|\bar{\sigma}_{i j l}(u)\right| d u \int_{0}^{+\infty}\left|\hat{\sigma}_{i j l}(u)\right| d u\left(z_{i}^{\prime}(t)\right)^{2} \\
& +\frac{1}{2} \sum_{i=1}^{n} \sum_{j=1}^{n} \sum_{l=1}^{n}\left|\alpha_{i} \gamma_{i}\right|\left|p_{i j l}(t)\right| M_{l}^{R} L_{j}^{R} \int_{0}^{+\infty}\left|\bar{\sigma}_{i j l}(u)\right| d u \int_{0}^{+\infty}\left|\hat{\sigma}_{i j l}(u)\right| d u z_{i}^{2}(t) \\
& +\frac{1}{2} \sum_{j=1}^{n} \sum_{i=1}^{n} \sum_{l=1}^{n}\left(\alpha_{j}^{2}+\left|\alpha_{j} \gamma_{j}\right|\right)\left|p_{j i l}(t)\right| M_{l}^{R} L_{i}^{R} \int_{0}^{+\infty}\left|\bar{\sigma}_{j i l}(u)\right| d u \\
& \times \int_{0}^{+\infty}\left|\hat{\sigma}_{j i l}(u)\right| z_{i}^{2}(t-u) d u,
\end{aligned}
$$

which, together with (2.4) and (2.5), entails

$$
W^{\prime}(t) \leq e^{2 \lambda t}\left\{\sum_{i=1}^{n}\left(\beta_{i}+\gamma_{i}^{2}+2 \lambda \alpha_{i} \gamma_{i}-a_{i}(t) \alpha_{i} \gamma_{i}-b_{i}(t) \alpha_{i}^{2}\right) z_{i}(t) z_{i}^{\prime}(t)\right.
$$




$$
\begin{aligned}
& +\sum_{i=1}^{n}\left[\lambda \alpha_{i}^{2}+\alpha_{i} \gamma_{i}-a_{i}(t) \alpha_{i}^{2}+\frac{1}{2} \alpha_{i}^{2} \sum_{j=1}^{n}\left(\left|c_{i j}(t)\right| L_{j}^{A}+\left|d_{i j}(t)\right| L_{j}^{B}\right)\right. \\
& +\frac{1}{2} \alpha_{i}^{2} \sum_{j=1}^{n} \sum_{l=1}^{n}\left|\theta_{i j l}(t)\right|\left(M_{j}^{Q} L_{l}^{Q}+L_{j}^{Q} M_{l}^{Q}\right) \\
& +\frac{1}{2} \alpha_{i}^{2} \sum_{j=1}^{n}\left|h_{i j}(t)\right| L_{j}^{K} \int_{0}^{+\infty}\left|\sigma_{i j}(u)\right| d u \\
& +\frac{1}{2} \alpha_{i}^{2} \sum_{j=1}^{n} \sum_{l=1}^{n}\left|p_{i j l}(t)\right| M_{j}^{R} L_{l}^{R} \int_{0}^{+\infty}\left|\hat{\sigma}_{i j l}(u)\right| d u \int_{0}^{+\infty}\left|\bar{\sigma}_{i j l}(u)\right| d u \\
& \left.+\frac{1}{2} \alpha_{i}^{2} \sum_{j=1}^{n} \sum_{l=1}^{n}\left|p_{i j l}(t)\right| M_{l}^{R} L_{j}^{R} \int_{0}^{+\infty}\left|\bar{\sigma}_{i j l}(u)\right| d u \int_{0}^{+\infty}\left|\hat{\sigma}_{i j l}(u)\right| d u\right]\left(z_{i}^{\prime}(t)\right)^{2} \\
& +\sum_{i=1}^{n}\left[-b_{i}(t) \alpha_{i} \gamma_{i}+\lambda \beta_{i}+\lambda \gamma_{i}^{2}+\frac{1}{2} \sum_{j=1}^{n}\left(\left|c_{i j}(t)\right| L_{j}^{A}+\left|d_{i j}(t)\right| L_{j}^{B}\right)\left|\alpha_{i} \gamma_{i}\right|\right. \\
& +\frac{1}{2} \sum_{j=1}^{n} \alpha_{j}^{2}\left(\left|c_{j i}(t)\right| L_{i}^{A}+d_{j i}^{+} L_{i}^{B} \frac{1}{1-\dot{q}_{j i}^{+}} e^{2 \lambda q_{j i}^{+}}\right) \\
& +\frac{1}{2} \sum_{j=1}^{n}\left(\left|c_{j i}(t)\right| L_{i}^{A}+d_{j i}^{+} L_{i}^{B} \frac{1}{1-\dot{q}_{j i}^{+}} e^{2 \lambda q_{j i}^{+}}\right)\left|\alpha_{j} \gamma_{j}\right| \\
& +\frac{1}{2} \sum_{j=1}^{n} \sum_{l=1}^{n}\left|\alpha_{i} \gamma_{i}\right|\left|\theta_{i j l}(t)\right|\left(M_{j}^{Q} L_{l}^{Q}+L_{j}^{Q} M_{l}^{Q}\right) \\
& +\frac{1}{2} \sum_{l=1}^{n} \sum_{j=1}^{n}\left(\alpha_{l}^{2}+\left|\alpha_{l} \gamma_{l}\right|\right) \theta_{l j i}^{+} M_{j}^{Q} L_{i}^{Q} e^{2 \lambda \xi_{l j i}^{+}} \frac{1}{1-\dot{\xi}_{l j i}^{+}} \\
& +\frac{1}{2} \sum_{j=1}^{n} \sum_{l=1}^{n}\left(\alpha_{j}^{2}+\left|\alpha_{j} \gamma_{j}\right|\right) \theta_{j i l}^{+} L_{i}^{Q} M_{l}^{Q} e^{2 \lambda \eta_{j i l}^{+}} \frac{1}{1-\dot{\eta}_{j i l}^{+}} \\
& +\frac{1}{2} \sum_{j=1}^{n}\left(\alpha_{j}^{2} h_{j i}^{+}+\left|\alpha_{j} \gamma_{j}\right| h_{j i}^{+}\right) L_{i}^{K} \int_{0}^{+\infty}\left|\sigma_{j i}(u)\right| e^{2 \lambda u} d u \\
& +\frac{1}{2} \sum_{l=1}^{n} \sum_{j=1}^{n}\left(\alpha_{l}^{2}+\left|\alpha_{l} \gamma_{l}\right|\right) p_{l j i}^{+} \int_{0}^{+\infty}\left|\hat{\sigma}_{l j i}(u)\right| d u \int_{0}^{+\infty}\left|\bar{\sigma}_{l j i}(u)\right| e^{2 \lambda u} d u M_{j}^{R} L_{i}^{R} \\
& +\frac{1}{2} \sum_{j=1}^{n} \sum_{l=1}^{n}\left(\alpha_{j}^{2}+\left|\alpha_{j} \gamma_{j}\right|\right) p_{j i l}^{+} \int_{0}^{+\infty}\left|\bar{\sigma}_{j i l}(u)\right| d u \int_{0}^{+\infty}\left|\hat{\sigma}_{j i l}(u)\right| e^{2 \lambda u} d u M_{l}^{R} L_{i}^{R} \\
& +\frac{1}{2} \sum_{j=1}^{n}\left|\alpha_{i} \gamma_{i}\right|\left|h_{i j}(t)\right| L_{j}^{K} \int_{0}^{+\infty}\left|\sigma_{i j}(u)\right| d u \\
& +\frac{1}{2} \sum_{j=1}^{n} \sum_{l=1}^{n}\left|\alpha_{i} \gamma_{i}\right|\left|p_{i j l}(t)\right| M_{j}^{R} L_{l}^{R} \int_{0}^{+\infty}\left|\hat{\sigma}_{i j l}(u)\right| d u \int_{0}^{+\infty}\left|\bar{\sigma}_{i j l}(u)\right| d u \\
& \left.\left.+\frac{1}{2} \sum_{j=1}^{n} \sum_{l=1}^{n}\left|\alpha_{i} \gamma_{i}\right|\left|p_{i j l}(t)\right| M_{l}^{R} L_{j}^{R} \int_{0}^{+\infty}\left|\bar{\sigma}_{i j l}(u)\right| d u \int_{0}^{+\infty}\left|\hat{\sigma}_{i j l}(u)\right| d u\right] z_{i}^{2}(t)\right\}
\end{aligned}
$$




$$
\begin{aligned}
& =e^{2 \lambda t}\left\{\sum_{i=1}^{n}\left(C_{i}^{\lambda}(t)\left(z_{i}^{\prime}(t)\right)^{2}+D_{i}^{\lambda}(t) z_{i}^{2}(t)+E_{i}^{\lambda}(t) z_{i}(t) z_{i}^{\prime}(t)\right)\right\} \\
& =e^{2 \lambda t}\left\{\sum_{i=1}^{n} C_{i}^{\lambda}(t)\left(z_{i}^{\prime}(t)+\frac{E_{i}^{\lambda}(t)}{2 C_{i}^{\lambda}(t)} z_{i}(t)\right)^{2}+\sum_{i=1}^{n}\left(D_{i}^{\lambda}(t)-\frac{\left(E_{i}^{\lambda}(t)\right)^{2}}{4 C_{i}^{\lambda}(t)}\right) z_{i}^{2}(t)\right\} \\
& \leq 0, \quad \forall t \in[0,+\infty) .
\end{aligned}
$$

This indicates that $W(t) \leq W(0)$ for all $t \in[0,+\infty)$, and

$$
\frac{1}{2} \sum_{i=1}^{n} \beta_{i} z_{i}^{2}(t) e^{2 \lambda t}+\frac{1}{2} \sum_{i=1}^{n}\left(\alpha_{i} z_{i}^{\prime}(t)+\gamma_{i} z_{i}(t)\right)^{2} e^{2 \lambda t} \leq W(0), \quad t \in[0,+\infty) .
$$

Manifestly,

$$
\left(\alpha_{i} z_{i}^{\prime}(t) e^{\lambda t}+\gamma_{i} z_{i}(t) e^{\lambda t}\right)^{2}=\left(\alpha_{i} z_{i}^{\prime}(t)+\gamma_{i} z_{i}(t)\right)^{2} e^{2 \lambda t}
$$

and

$$
\alpha_{i}\left|z_{i}^{\prime}(t)\right| e^{\lambda t} \leq\left|\alpha_{i} z_{i}^{\prime}(t) e^{\lambda t}+\gamma_{i} z_{i}(t) e^{\lambda t}\right|+\left|\gamma_{i} z_{i}(t) e^{\lambda t}\right| .
$$

Combining with the Cauchy-Schwarz inequality, one can pick a constant $M>0$ such that

$$
\left|z_{i}^{\prime}(t)\right| \leq M e^{-\lambda t}, \quad\left|z_{i}(t)\right| \leq M e^{-\lambda t}, \quad t \geq 0, i \in D,
$$

which proves Lemma 2.1.

Remark 2.2 More precisely, according to Lemma 2.1, we know that, if $y(t)$ is an equilibrium point or a $T$-anti-periodic solution of (1.1), then all solutions of the system (1.1) and their derivatives are exponentially convergent to $y(t)$ and $y^{\prime}(t)$, respectively. Referring to the definition of stability adopted in $[5,18-21,40,42-45]$, this indicates that $y(t)$ is globally exponentially stable.

\section{Anti-periodicity of HIHNNs (1.1)}

Now, we set out to present the main result of this paper as follows.

Theorem 3.1 Under conditions $\left(G_{1}\right)-\left(G_{4}\right)$, system (1.1) has a globally exponentially stable T-anti-periodic solution.

Proof Let $v(t)=\left(v_{1}(t), v_{2}(t), \ldots, v_{n}(t)\right)$ be a solution of system (1.1) with initial conditions:

$$
v_{i}(s)=\varphi_{i}^{v}(s), \quad v_{i}^{\prime}(0)=\psi_{i}^{v}, \quad \varphi_{i}^{\nu} \in B C((-\infty, 0], \mathbb{R}), \psi_{i}^{v} \in \mathbb{R}, i \in D .
$$

Clearly, for any nonnegative integer $m$,

$$
\begin{aligned}
& \left((-1)^{m+1} v_{i}(t+(m+1) T)\right)^{\prime \prime} \\
& \quad=-a_{i}(t)\left((-1)^{m+1} v_{i}(t+(m+1) T)\right)^{\prime}-b_{i}(t)\left((-1)^{m+1} v_{i}(t+(m+1) T)\right)
\end{aligned}
$$




$$
\begin{aligned}
& +\sum_{j=1}^{n} c_{i j}(t) A_{j}\left((-1)^{m+1} v_{j}(t+(m+1) T)\right) \\
& +\sum_{j=1}^{n} d_{i j}(t) B_{j}\left((-1)^{m+1} v_{j}\left(t+(m+1) T-q_{i j}(t)\right)\right)+\sum_{j=1}^{n} \sum_{l=1}^{n} \theta_{i j l}(t) \\
& \times Q_{j}\left((-1)^{m+1} v_{j}\left(t+(m+1) T-\eta_{i j l}(t)\right)\right) \\
& \times Q_{l}\left((-1)^{m+1} v_{l}\left(t+(m+1) T-\xi_{i j l}(t)\right)\right) \\
& +\sum_{j=1}^{n} h_{i j}(t) \int_{0}^{+\infty} \sigma_{i j}(u) K_{j}\left((-1)^{m+1} v_{j}(t-u)\right) d u \\
& +\sum_{j=1}^{n} \sum_{l=1}^{n} p_{i j l}(t) \int_{0}^{+\infty} \hat{\sigma}_{i j l}(u) R_{j}\left((-1)^{m+1} v_{j}(t-u)\right) d u \\
& \times \int_{0}^{+\infty} \bar{\sigma}_{i j l}(u) R_{l}\left((-1)^{m+1} v_{l}(t-u)\right) d u+J_{i}(t)
\end{aligned}
$$

for all $i \in D, t+(m+1) T \geq 0$. It is easy to see that $(-1)^{m+1} v(t+(m+1) T)$ is a solution of (1.1), and $u(t)=-v(t+T)$ satisfies system (1.1) involving initial values:

$$
\varphi_{i}^{u}(s)=-v_{i}(s+T), \quad \psi_{i}^{u}=-v_{i}^{\prime}(T), \quad \text { for all } s \in(-\infty, 0], i \in D
$$

According to Lemma 2.1, we can take a constant $N=N\left(\varphi^{v}, \psi^{v}, \varphi^{u}, \psi^{u}\right)$ satisfying

$$
\left|v_{i}(t)-u_{i}(t)\right| \leq N e^{-\lambda t}, \quad\left|v_{i}^{\prime}(t)-u_{i}^{\prime}(t)\right| \leq N e^{-\lambda t}, \quad \text { for all } t \geq 0, i \in D
$$

Thus,

$$
\left.\begin{array}{c}
\left|(-1)^{p} v_{i}(t+p T)-(-1)^{p+1} v_{i}(t+(p+1) T)\right| \\
\quad=\left|v_{i}(t+p T)-u_{i}(t+p T)\right| \leq N e^{-\lambda(t+p T)}, \\
\left|\left((-1)^{p} v_{i}(t+p T)\right)^{\prime}-\left((-1)^{p+1} v_{i}(t+(p+1) T)\right)^{\prime}\right| \\
\quad=\left|v_{i}^{\prime}(t+p T)-u_{i}^{\prime}(t+p T)\right| \leq N e^{-\lambda(t+p T)},
\end{array}\right\} \quad \forall i \in D, t+p T \geq 0
$$

Thus, together with the facts that

$$
\begin{aligned}
& (-1)^{m+1} v_{i}(t+(m+1) T) \\
& =v_{i}(t)+\sum_{p=0}^{m}\left[(-1)^{p+1} v_{i}(t+(p+1) T)-(-1)^{p} v_{i}(t+p T)\right] \quad(i \in D)
\end{aligned}
$$

and

$$
\begin{aligned}
& \left((-1)^{m+1} v_{i}(t+(m+1) T)\right)^{\prime} \\
& \quad=v_{i}^{\prime}(t)+\sum_{p=0}^{m}\left[\left((-1)^{p+1} v_{i}(t+(p+1) T)\right)^{\prime}-\left((-1)^{p+1} v_{i}(t+p T)\right)^{\prime}\right] \quad(i \in D),
\end{aligned}
$$


then, we can show that there exists a continuous differentiable function $\kappa(t)=\left(\kappa_{1}(t), \kappa_{2}(t)\right.$, $\left.\ldots, \kappa_{n}(t)\right)$ such that $\left\{(-1)^{m} v(t+m T)\right\}_{m \geq 1}$ and $\left\{\left((-1)^{m} v(t+m T)\right)^{\prime}\right\}_{m \geq 1}$ are uniformly convergent to $\kappa(t)$ and $\kappa^{\prime}(t)$ on any compact set of $\mathbb{R}$, respectively. Moreover,

$$
\begin{aligned}
\kappa(t+T) & =\lim _{m \rightarrow+\infty}(-1)^{m} v(t+T+m T) \\
& =-\lim _{(m+1) \rightarrow+\infty}(-1)^{m+1} v(t+(m+1) T)=-\kappa(t)
\end{aligned}
$$

involves that $\kappa(t)$ is $T$-anti-periodic on $\mathbb{R}$. It follows from $\left(G_{1}\right)-\left(G_{4}\right)$ and the continuity on (3.2) that $\left\{v^{\prime \prime}(t+(m+1) T)\right\}_{m \geq 1}$ uniformly converges to a continuous function on any compact set of $\mathbb{R}$. Furthermore, for any compact set of $\mathbb{R}$, setting $m \longrightarrow+\infty$, we obtain

$$
\begin{aligned}
\kappa_{i}^{\prime \prime}(t)= & -a_{i}(t) \kappa_{i}^{\prime}(t)-b_{i}(t) \kappa_{i}(t)+\sum_{j=1}^{n} c_{i j}(t) A_{j}\left(\kappa_{j}(t)\right) \\
& +\sum_{j=1}^{n} d_{i j}(t) B_{j}\left(\kappa_{j}\left(t-q_{i j}(t)\right)\right) \\
& +\sum_{j=1}^{n} \sum_{l=1}^{n} \theta_{i j l}(t) Q_{j}\left(\kappa_{j}\left(t-\eta_{i j l}(t)\right)\right) Q_{l}\left(\kappa_{l}\left(t-\xi_{i j l}(t)\right)\right) \\
& +\sum_{j=1}^{n} h_{i j}(t) \int_{0}^{+\infty} \sigma_{i j}(u) K_{j}\left(\kappa_{j}(t-u)\right) d u \\
& +\sum_{j=1}^{n} \sum_{l=1}^{n} p_{i j l}(t) \int_{0}^{+\infty} \hat{\sigma}_{i j l}(u) R_{j}\left(\kappa_{j}(t-u)\right) d u \\
& \times \int_{0}^{+\infty} \bar{\sigma}_{i j l}(u) R_{l}\left(\kappa_{l}(t-u)\right) d u+J_{i}(t), \quad i \in D,
\end{aligned}
$$

which involves the fact that $\kappa(t)$ is a $T$-anti-periodic solution of (1.1). With the aid of Lemma 2.1 and Remark 2.2, we find that $\kappa(t)$ is globally exponentially stable. This completes the proof of Theorem 3.1.

Remark 3.1 In this present paper, the exponential convergence on every solution and its derivative in system (1.1) is established. In particular, one can see that the results in [19$21,30,34]$ are a special case of Theorem 3.1. This indicates that our results generalize and improve the previous references.

\section{A numerical example}

In this section, we give an example with a simulation to demonstrate the feasibility and the validity of our theoretical results. 
Example 4.1 Let $n=2$, and let us regard the following high-order inertial Hopfield neural networks with mixed delays

$$
\begin{aligned}
x_{1}^{\prime \prime}(t)= & -(14.92+0.1|\sin t|) x_{1}^{\prime}(t)-(27.89+0.2|\sin t|) x_{1}(t) \\
& +2.28(\sin t) A_{1}\left(x_{1}(t)\right)+2.19(\cos t) A_{2}\left(x_{2}(t)\right) \\
& -0.84(\cos 2 t) B_{1}\left(x_{1}\left(t-0.2 \sin ^{2} t\right)\right)+2.41(\cos 2 t) B_{2}\left(x_{2}\left(t-0.3 \sin ^{2} t\right)\right) \\
& +4(\sin t) Q_{1}\left(x_{1}\left(t-0.4 \sin ^{2} t\right)\right) Q_{2}\left(x_{2}\left(t-0.5 \sin ^{2} t\right)\right) \\
& -0.95(\cos 2 t) \int_{0}^{+\infty} \sin (4 u) e^{-0.5 u} K_{1}\left(x_{1}(t-u)\right) d u \\
& +2.52(\sin 2 t) \int_{0}^{+\infty} \sin (3 u) e^{-u} K_{2}\left(x_{2}(t-u)\right) d u \\
& +3.8(\cos t) \int_{0}^{+\infty} \cos (2 u) e^{-0.5 u} R_{1}\left(x_{1}(t-u)\right) d u \\
& \times \int_{0}^{+\infty} \cos (2 u) e^{-u} R_{2}\left(x_{2}(t-u)\right) d u+55 \sin t, \\
x_{2}^{\prime \prime}(t)= & \left.-(15.11+0.1|\cos t|) x_{2}^{\prime}(t)-(31.05-0.1|\sin t|)\right) x_{2}(t) \\
& -1.88(\sin t) A_{1}\left(x_{1}(t)\right)-2.33(\cos t) A_{2}\left(x_{2}(t)\right) \\
& -2.18(\sin 2 t) B_{1}\left(x_{1}\left(t-0.2 \cos ^{2} t\right)\right)+3.18(\sin 2 t) B_{2}\left(x_{2}\left(t-0.3 \cos ^{2} t\right)\right) \\
& +3.8(\sin t) Q_{1}\left(x_{1}\left(t-0.4 \cos ^{2} t\right)\right) Q_{2}\left(x_{2}\left(t-0.5 \cos ^{2} t\right)\right) \\
& -2.28(\cos 2 t) \int_{0}^{+\infty} \sin (2 u) e^{-0.5 u} K_{1}\left(x_{1}(t-u)\right) d u \\
& +3.28(\sin 2 t) \int_{0}^{+\infty} \sin (5 u) e^{-u} K_{2}\left(x_{2}(t-u)\right) d u \\
& +4(\cos t) \int_{0}^{+\infty} \cos (4 u) e^{-0.5 u} R_{1}\left(x_{1}(t-u)\right) d u \\
& \times \int_{0}^{+\infty} \cos (4 u) e^{-u} R_{2}\left(x_{2}(t-u)\right) d u+48 \sin t,
\end{aligned}
$$

where $A_{1}(u)=A_{2}(u)=\frac{1}{35}|u|, B_{1}(u)=B_{2}(u)=\frac{1}{48} u, Q_{1}(u)=Q_{2}(u)=\frac{1}{110}(|u+1|-|u-1|)$, $K_{1}(u)=K_{2}(u)=\frac{1}{4 \pi} \arctan u, R_{1}(u)=R_{2}(u)=\frac{1}{2 \pi}|\arctan u|$.

Obviously, one can take $\lambda=0.01, \alpha_{i}=\gamma_{i}=1.1, \beta_{i}=5, L_{i}^{A}=\frac{1}{35}, L_{i}^{B}=\frac{1}{48}, L_{i}^{Q}=\frac{1}{55}, L_{i}^{K}=L_{i}^{R}=$ $\frac{1}{2}, M_{i}^{Q}=\frac{1}{55}, M_{i}^{R}=\frac{1}{4}, i=1,2$, such that (4.1) satisfies (2.4) and all the conditions assumed in Sect. 2. By Theorem 3.1, we know that system (4.1) has a globally exponentially stable $\pi$ anti-periodic solution $x^{*}(t)$, and every solution of (4.1) and its derivative are exponentially convergent to $x^{*}(t)$ and $\left(x^{*}(t)\right)^{\prime}$, respectively. Simulations in Figs. 1 and 2 reflect that the theoretical periodicity is in agreement with the numerically observed behavior.

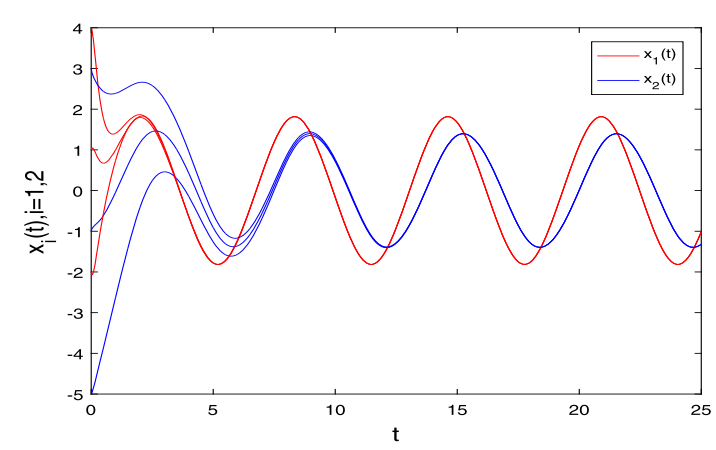

Figure 1 Numerical solutions $x(t)$ to system (4.1) with initial values: $\left(\varphi_{1}(s), \varphi_{2}(s), \psi_{1}(s), \psi_{2}(s)\right) \equiv(2 \sin t+1$, $-2 \cos t-3,2,0),(2 \cos t+2,3 \sin t-1,0,3),(-3 \sin t-2,-4 \sin t+3,-3,-4)$ 


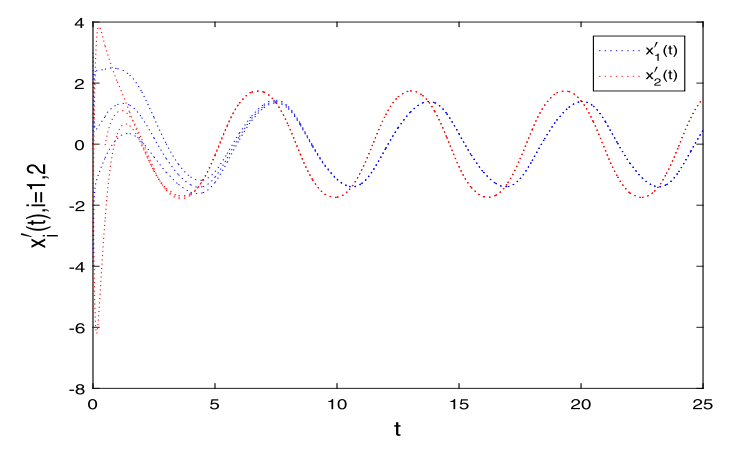

Figure 2 Numerical solutions $x^{\prime}(t)$ to system (4.1) with initial value $\left(\varphi_{1}(s), \varphi_{2}(s), \psi_{1}(s), \psi_{2}(s)\right) \equiv(2 \sin t+1$, $-2 \cos t-3,2,0),(2 \cos t+2,3 \sin t-1,0,3),(-3 \sin t-2,-4 \sin t+3,-3,-4)$

Remark 4.1 By the way, there are many excellent results on inertial Hopfield neural networks with time-varying delays [18-21, 27, 29, 30]. However, the anti-periodicity on high-order inertial Hopfield neural networks with bounded time-varying delays and unbounded continuously distributed delays have never been touched upon by using the nonreduced order method. In addition, the corresponding results of [17-21, 27, 29, 30] and [46-95] cannot be used to reveal the convergence of the anti-periodic solution of the system (4.1).

\section{Conclusions}

In this paper, abandoning the traditional reduced order method, we explore the global convergence dynamics on a class of anti-periodic high-order inertial Hopfield neural networks with bounded time-varying delays and unbounded continuously distributed delays. Some sufficient conditions have been obtained to guarantee that every solution and its derivative of the addressed model is exponentially convergent to a anti-periodic solution and its derivative by combining differential inequality techniques with the Lyapunov function method. It should be mentioned that the results obtained in this manuscript are novel, and the method adopted provides a possible effective approach for studying other types high-order inertial neural networks with mixed delays.

Acknowledgements

The authors would like to thank the anonymous referees and the editor for very helpful suggestions and comments which led to improvements of the original paper.

\section{Funding}

This work was supported by Natural Science Foundation of Hunan Province of China (Nos. 2019JJ40141, 2019JJ40142), and Scientific Research Fund of Hunan Provincial Education Department (19A267).

Availability of data and materials

Data sharing not applicable to this article as no datasets were generated or analyzed during the current study.

Competing interests

The authors declare that they have no competing interests.

Authors' contributions

The two authors contributed equally to this work. All authors read and approved the final manuscript.

\section{Author details}

${ }^{1}$ Key Laboratory of Hunan Province for Statistical Learning and Intelligent Computation; School of Mathematics and Statistics, Hunan University of Technology and Business, 410205 Changsha, Hunan, P.R. China. ${ }^{2}$ College of Mathematics and Physics, Hunan University of Arts and Science, 415000 Changde, Hunan, P.R. China. 


\section{Publisher's Note}

Springer Nature remains neutral with regard to jurisdictional claims in published maps and institutional affiliations.

Received: 16 April 2020 Accepted: 17 June 2020 Published online: 02 July 2020

\section{References}

1. Babcock, K., Westervelt, R.: Stability and dynamics of simple electronic neural networks with added inertia. Physica D 23, 464-469 (1986)

2. Babcock, K., Westervelt, R.: Dynamics of simple electronic neural networks. Physica D 28, 305-316 (1987)

3. Yu, S., Zhang, Z., Quan, Z.: New global exponential stability conditions for inertial Cohen-Grossberg neural networks with time delays. Neurocomputing 151, 1446-1454 (2015)

4. Cai, Z., Huang, J., Huang, L.: Periodic orbit analysis for the delayed Filippov system. Proc. Am. Math. Soc. 146 4667-4682 (2018)

5. Huang, C.: Exponential stability of inertial neural networks involving proportional delays and non-reduced order method. J. Exp. Theor. Artif. Intell. 32(1), 133-146 (2020)

6. Li, C., Chen, G., Liao, L., Yu, J.: Hopf bifurcation and chaos in a single inertial neuron model with time delay. Eur. Phys. J. B 41, 337-343 (2004)

7. Zhao, H., Yu, X., Wang, L.: Bifurcation and control in an inertial two-neuron system with time delays. Int. J. Bifurc. Chaos 22(2), $1250036(2012)$

8. Wang, J., Tian, L.: Global Lagrange stability for inertial neural networks with mixed time varying delays. Neurocomputing 235, 140-146 (2017)

9. $\mathrm{Ge}, \mathrm{J} ., \mathrm{Xu}, \mathrm{J}$.: Weak resonant double Hopf bifurcations in an inertial four-neuron model with time delay. Int. J. Neural Syst. 22, 63-75 (2012)

10. Zhang, H., Cao, Q., Yang, H.: Asymptotically almost periodic dynamics on delayed Nicholson-type system involving patch structure. J. Inequal. Appl. 2020, 102 (2020)

11. Chen, T., Huang, L., Yu, P., Huang, W.: Bifurcation of limit cycles at infinity in piecewise polynomial systems. Nonlinear Anal., Real World Appl. 41, 82-106 (2018)

12. Li, X., Li, X., Hu, C.: Some new results on stability and synchronization for delayed inertial neural networks based on non-reduced order method. Neural Netw. 96, 91-100 (2017)

13. Yang, X., Wen, S., Liu, Z., Li, C., Huang, C.: Dynamic properties of foreign exchange complex network. Mathematics 7, 832 (2019). https://doi.org/10.3390/math7090832

14. Huang, C., Yang, L., Cao, J.: Asymptotic behavior for a class of population dynamics. AlMS Math. 54(4), 3378-3390 (2020). https://doi.org/10.3934/math.2020218

15. Ke, Y., Miao, C.: Stability and existence of periodic solutions in inertial BAM neural networks with time delay. Neural Comput. Appl. 23, 1089-1099 (2013)

16. Ke, Y., Miao, C.: Anti-periodic solutions of inertial neural networks with time delays. Neural Process. Lett. 45, 523-538 (2017)

17. Xu, C., Zhang, Q.: Existence and global exponential stability of anti-periodic solutions for BAM neural networks with inertial term and delay. Neurocomputing 153, 108-116 (2015)

18. Huang, C., Liu, B.: New studies on dynamic analysis of inertial neural networks involving non-reduced order method. Neurocomputing 325, 283-287 (2019)

19. Huang, C., Yang, L., Liu, B.: New results on periodicity of non-autonomous inertial neural networks involving non-reduced order method. Neural Process. Lett. 50, 595-606 (2019)

20. Huang, C., Zhang, H.: Periodicity of non-autonomous inertial neural networks involving proportional delays and non-reduced order method. Int. J. Biomath. 12(2), 1-13 (2019)

21. Zhang, J., Huang, C.: Dynamics analysis on a class of delayed neural networks involving inertial terms. Adv. Differ. Equ. 2020, 120 (2020). https://doi.org/10.1186/s13662-020-02566-4

22. Belley, J.-M., Bondo, E.: Anti-periodic solutions of Liénard equations with state dependent impulses. J. Differ. Equ. 261(7), 4164-4187 (2016)

23. Peng, J., Zhang, Y.: Heron triangles with figurate number sides. Acta Math. Hung. 157(2), 478-488 (2019)

24. Huang, C., Wang, J., Huang, L.: Asymptotically almost periodicity of delayed Nicholson-type system involving patch structure. Electron. J. Differ. Equ. 2020, 61 (2020)

25. Huang, C., Wen, S., Huang, L.: Dynamics of anti-periodic solutions on shunting inhibitory cellular neural networks with multi-proportional delays. Neurocomputing 357, 47-52 (2019)

26. Huang, C., Long, X., Cao, J.: Stability of antiperiodic recurrent neural networks with multiproportional delays. Math. Methods Appl. Sci. 43(9), 6093-6102 (2020). https://doi.org/10.1002/mma.6350

27. Li, Y., Meng, X., Xiong, L.: Pseudo almost periodic solutions for neutral type high-order Hopfield neural networks with mixed time-varying delays and leakage delays on time scales. Int. J. Mach. Learn. Cybern. 8(6), 1-13 (2017)

28. Li, J., Ying, J., Xie, D.: On the analysis and application of an ion size-modified Poisson-Boltzmann equation. Nonlinear Anal., Real World Appl. 47, 188-203 (2019)

29. Xiao, B., Meng, H.: Existence and exponential stability of positive almost periodic solutions for high-order Hopfield neural networks. Appl. Math. Mech. 33, 532-542 (2009)

30. Yao, L.: Global exponential stability on anti-periodic solutions in proportional delayed HIHNNs. J. Exp. Theor. Artif. Intell. (2020). https://doi.org/10.1080/0952813X.2020.1721571

31. $\mathrm{Xu}, \mathrm{Y}$ :: Exponential stability of weighted pseudo almost periodic solutions for HCNNs with mixed delays. Neural Process. Lett. 46(2), 507-519 (2017)

32. Huang, C., Liu, B., Tian, X.: Global convergence on asymptotically almost periodic SICNNs with nonlinear decay functions. Neural Process. Lett. 49, 625-641 (2019)

33. Huang, C., Kuang, H., Chen, X., Wen, F.: An LMl approach for dynamics of switched cellular neural networks with mixed delays. Abstr. Appl. Anal. 2013, 870486 (2013). https://doi.org/10.1155/2013/870486

34. $\mathrm{Xu}, \mathrm{Y}$.: Convergence on non-autonomous inertial neural networks with unbounded distributed delays. J. Exp. Theor. Artif. Intell. 32(3), 503-513 (2020). https://doi.org/10.1080/0952813X.2019.1652941 
35. Zhao, C., Wang, Z.: Exponential convergence of a SICNN with leakage delays and continuously distributed delays of neutral type. Neural Process. Lett. 41, 239-247 (2015)

36. Li, W., Huang, L., Ji, J.: Periodic solution and its stability of a delayed Beddington-Deangelis type predator-prey system with discontinuous control strategy. Math. Methods Appl. Sci. 42(13), 4498-4515 (2019)

37. Iswarya, M., Raja, R., Rajchakit, G., Cao, J., Alzabut, J., Huang, C.: Existence, uniqueness and exponential stability of periodic solution for discrete-time delayed BAM neural networks based on coincidence degree theory and graph theoretic method. Mathematics 7(11), 1055 (2019). https://doi.org/10.3390/math7111055

38. Zhao, J., Liu, J., Fang, L.: Anti-periodic boundary value problems of second-order functional differential equations. Bull. Malays. Math. Sci. Soc. 37(2), 311-320 (2014)

39. Xu, Y., Cao, Q., Guo, X.: Stability on a patch structure Nicholson's blowflies system involving distinctive delays. Appl. Math. Lett. 105, 106340 (2020). https://doi.org/10.1016/j.aml.2020.106340

40. Ke, Y., Miao, C.: Stability analysis of inertial Cohen-Grossberg-type neural networks with time delays. Neurocomputing 117, 196-205 (2013)

41. Hino, Y., Murakami, S., Naito, T.: Functional Differential Equations with Infinite Delay. Lecture in Mathematics, vol. 1473. Springer, Berlin (1991)

42. Duan, L., Huang, C.: Existence and global attractivity of almost periodic solutions for a delayed differential neoclassical growth model. Math. Methods Appl. Sci. 40(3), 814-822 (2017)

43. Huang, C., Cao, J., Cao, J.: Stability analysis of switched cellular neural networks: a mode-dependent average dwell time approach. Neural Netw. 82, 84-99 (2016). https://doi.org/10.1016/j.neunet.2016.07.009

44. Liu, B.: Finite-time stability of CNNs with neutral proportional delays and time-varying leakage delays. Math. Methods Appl. Sci. 40, 167-174 (2017)

45. Huang, C., Yang, H., Cao, J.: Weighted pseudo almost periodicity of multi-proportional delayed shunting inhibitory cellular neural networks with D operator. Discrete Contin. Dyn. Syst., Ser. S (2020). https://doi.org/10.3934/dcdss.2020372

46. Huang, C., Yang, Z., Yi, T., Zou, X.: On the basins of attraction for a class of delay differential equations with non-monotone bistable nonlinearities. J. Differ. Equ. 256(7), 2101-2114 (2014). https://doi.org/10.1016/j.jde.2013.12.015

47. Huang, C., Long, X., Huang, L., Fu, S.: Stability of almost periodic Nicholson's blowflies model involving patch structure and mortality terms. Canad. Math. Bull., 63(2), 405-422 (2020). https://doi.org/10.4153/S0008439519000511

48. Huang, C., Zhang, H., Huang, L.: Almost periodicity analysis for a delayed Nicholson's blowflies model with nonlinear density-dependent mortality term. Commun. Pure Appl. Anal. 18(6), 3337-3349 (2019)

49. Hu, H., Yi, T., Zou, X.: On spatial-temporal dynamics of a Fisher-KPP equation with a shifting environment. Proc. Am. Math. Soc. 148, 213-221 (2020)

50. Hu, H., Yuan, X., Huang, L., Huang, C.: Global dynamics of an SIRS model with demographics and transfer from infectious to susceptible on heterogeneous networks. Math. Biosci. Eng. 16(5), 5729-5749 (2019)

51. Hu, H., Zou, X.: Existence of an extinction wave in the Fisher equation with a shifting habitat. Proc. Am. Math. Soc. 145(11), 4763-4771 (2017)

52. Wang, J., Huang, C., Huang, L:: Discontinuity-induced limit cycles in a general planar piecewise linear system of saddle-focus type. Nonlinear Anal. Hybrid Syst. 33, 162-178 (2019)

53. Wang, J., Chen, X., Huang, L.: The number and stability of limit cycles for planar piecewise linear systems of node-saddle type. J. Math. Anal. Appl. 469(1), 405-427 (2019)

54. Zhang, H., Qian, C.: Convergence analysis on inertial proportional delayed neural networks. Adv. Differ. Equ. (2020). https://doi.org/10.1186/s13662-020-02737-3

55. Qian, C.: New periodic stability for a class of Nicholson's blowflies models with multiple different delays. Int. J. Control (2020). https://doi.org/10.1080/00207179.2020.1766118

56. Huang, C., Su, R., Cao, J., Xiao, S.: Asymptotically stable high-order neutral cellular neural networks with proportional delays and D operators. Math. Comput. Simul. 107, 127-135 (2020). https://doi.org/10.1016/j.matcom.2019.06.001

57. Zhang, $\mathrm{X} ., \mathrm{Hu}, \mathrm{H}$.: Convergence in a system of critical neutral functional differential equations. Appl. Math. Lett. 107, 106385 (2020). https://doi.org/10.1016/j.aml.2020.106385

58. Tan, Y., Huang, C., Sun, B., Wang, T.: Dynamics of a class of delayed reaction-diffusion systems with Neumann boundary condition. J. Math. Anal. Appl. 458(2), 1115-1130 (2018)

59. Huang, C., Yang, X., Cao, J.: Stability analysis of Nicholson's blowflies equation with two different delays. Math. Comput. Simul. 171, 201-206 (2020). https://doi.org/10.1016/.matcom.2019.09.023

60. Zhang, Y:: Some observations on the Diophantine equation $f(x) f(y)=f(z)^{2}$. Colloq. Math. 142(2), 275-283 (2016)

61. Li, L., Jin, Q., Yao, B.: Regularity of fuzzy convergence spaces. Open Math. 16, 1455-1465 (2018)

62. Lv, B., Huang, L., Wang, K.: Endomorphisms of twisted Grassmann graphs. Graphs Comb. 33(1), 157-169 (2017)

63. Huang, C., Peng, C., Chen, X., Wen, F.: Dynamics analysis of a class of delayed economic model. Abstr. Appl. Anal. 2013, 962738 (2013). https://doi.org/10.1155/2013/962738

64. Zhang, Y: Right triangle and parallelogram pairs with a common area and a common perimeter. J. Number Theory $164,179-190(2016)$

65. Huang, L., Su, H., Tang, G., Wang, J.: Bilinear forms graphs over residue class rings. Linear Algebra Appl. 523, 13-32 (2017)

66. Cao, Q., Guo, X.: Anti-periodic dynamics on high-order inertial Hopfield neural networks involving time-varying delays. AIMS Math. 5(6), 5402-5421 (2020). https://doi.org/10.3934/math.2020347

67. Li, X., Liu, Y., Wu, J.: Flocking and pattern motion in a modified Cucker-Smale model. Bull. Korean Math. Soc. 53(5), $1327-1339(2016)$

68. Xie, Y., Li, Q., Zhu, K.: Attractors for nonclassical diffusion equations with arbitrary polynomial growth nonlinearity. Nonlinear Anal., Real World Appl. 31, 23-37 (2016)

69. Xie, Y., Li, Y., Zeng, Y.: Uniform attractors for nonclassical diffusion equations with memory. J. Funct. Spaces 2016 5340489 (2016). https://doi.org/10.1155/2016/5340489

70. Wang, F., Wang, P., Yao, Z.: Approximate controllability of fractional partial differential equation. Adv. Differ. Equ. 2015, 367 (2015). https://doi.org/10.1186/s13662-015-0692-3 
71. Liu, Y., Wu, J.: Multiple solutions of ordinary differential systems with min-max terms and applications to the fuzzy differential equations. Adv. Differ. Equ. 2015, 379 (2015). https://doi.org/10.1186/s13662-015-0708-z

72. Yan, L., Liu, J., Luo, Z.: Existence and multiplicity of solutions for second-order impulsive differential equations on the half-line. Adv. Differ. Equ. 2013, 293 (2013). https://doi.org/10.1186/1687-1847-2013-293

73. Huang, L., LV, B.: Cores and independence numbers of Grassmann graphs. Graphs Comb. 33(6), 1607-1620 (2017)

74. Liu, W.: An incremental approach to obtaining attribute reduction for dynamic decision systems. Open Math. 14, $875-888(2016)$

75. Huang, C., Zhang, H., Cao, J., Hu, H.: Stability and Hopf bifurcation of a delayed prey-predator model with disease in the predator. Int. J. Bifurc. Chaos 29(7), 1-23 (2019). https://doi.org/10.1142/S0218127419500913

76. Wang, F., Yao, Z:: Approximate controllability of fractional neutral differential systems with bounded delay. Fixed Point Theory 17, 495-508 (2016)

77. Zhou, S., Jiang, Y.: Finite volume methods for N-dimensional time fractional Fokker-Planck equations. Bull. Malays. Math. Sci. Soc. 42(6), 3167-3186 (2019)

78. Huang, C., Wen, S. Li, M., Wen, F., Yang, X.: An empirical evaluation of the influential nodes for stock market network: Chinese A shares case. Finance Res. Lett. (2020). https://doi.org/10.1016/j.frl.2020.101517

79. Liu, F., Feng, L., Vo, A., Li, J.: Unstructured-mesh Galerkin finite element method for the two-dimensional multi-term time-space fractional Bloch-Torrey equations on irregular convex domains. Comput. Math. Appl. 78(5), 1637-1650 (2019)

80. Jin, Q., Li, L., Lang, G.: -Regularity and p-regular modification in T-convergence spaces. Mathematics, 7(4), 370 (2019). https://doi.org/10.3390/math7040370

81. Huang, L.: Endomorphisms and cores of quadratic forms graphs in odd characteristic. Finite Fields Appl. 55, 284-304 (2019)

82. Huang, L., Lv, B., Wang, K.: Erdos-Ko-Rado theorem, Grassmann graphs and $p^{5}$-Kneser graphs for vector spaces over a residue class ring. J. Comb. Theory, Ser. A 164, 125-158 (2019)

83. Li, Y., Vuorinen, M., Zhou, Q.: Characterizations of John spaces. Monatshefte Math. 188(3), 547-559 (2019)

84. Huang, L., LV, B., Wang, K.: The endomorphisms of Grassmann graphs. Ars Math. Contemp. 10(2), 383-392 (2016)

85. Gong, X., Wen, F., He, Z., Yang, J., Yang, X.: Extreme return, extreme volatility and investor sentiment. Filomat 30(15), 3949-3961 (2016)

86. Jiang, Y., Huang, B.: A note on the value distribution of $f^{1}\left(f^{(k)}\right)^{n}$. Hiroshima Math. J. 46(2), 135-147 (2016)

87. Huang, L., Huang, J., Zhao, K.: On endomorphisms of alternating forms graph. Discrete Math. 338(3), 110-121 (2015)

88. Huang, C., Qiao, Y., Huang, L., Agarwal, R.P.: Dynamical behaviors of a food-chain model with stage structure and time delays. Adv. Differ. Equ. 2018, 186 (2018). https://doi.org/10.1186/s13662-018-1589-8

89. Huang, C., Cao, J., Wen, F., Yang, X.: Stability analysis of SIR model with distributed delay on complex networks. PLoS ONE 11(8), e0158813 (2016). https://doi.org/10.1371/journal.pone.0158813

90. Zhou, Y., Wan, X., Huang, C., Yang, X.: Finite-time stochastic synchronization of dynamic networks with nonlinear coupling strength via quantized intermittent control. Appl. Math. Comput. 376, 125157 (2020). https://doi.org/10.1016/j.amc.2020.125157

91. Wang, W., Huang, C., Huang, C., Cao, J., Lu, J., Wang, L.: Bipartite formation problem of second-order nonlinear multi-agent systems with hybrid impulses. Appl. Math. Comput. 370, 124926 (2020). https://doi.org/10.1016/j.amc.2019.124926

92. Wang, W., Liu, F., Chen, W.: Exponential stability of pseudo almost periodic delayed Nicholson-type system with patch structure. Math. Methods Appl. Sci. 42(2), 592-604 (2019)

93. Wang, W.: Finite-time synchronization for a class of fuzzy cellular neural networks with time-varying coefficients and proportional delays. Fuzzy Sets Syst. 338, 40-49 (2018)

94. Wang, W., Shi, C., Chen, W.: Stochastic Nicholson-type delay differential system. Int. J. Control, 1-14 (2019). https://doi.org/10.1080/00207179.2019.1651941

95. Qian, C., Hu, Y.: Novel stability criteria on nonlinear density-dependent mortality Nicholson's blowflies systems in asymptotically almost periodic environments. J. Inequal. Appl. (2020). https://doi.org/10.1186/s13660-019-2275-4

\section{Submit your manuscript to a SpringerOpen ${ }^{\circ}$ journal and benefit from:}

- Convenient online submission

- Rigorous peer review

- Open access: articles freely available online

- High visibility within the field

- Retaining the copyright to your article

Submit your next manuscript at $\boldsymbol{\nabla}$ springeropen.com 